\title{
Stable scalable control of soliton propagation in broadband nonlinear optical waveguides
}

\author{
Avner Peleg ${ }^{1}$, Quan M. Nguyen ${ }^{2}$, and Toan T. Huynh ${ }^{3}$ \\ ${ }^{1}$ Department of Exact Sciences, Afeka College of Engineering, Tel Aviv 69988, Israel \\ ${ }^{2}$ Department of Mathematics, International University, \\ Vietnam National University-HCMC, Ho Chi Minh City, Vietnam and \\ ${ }^{3}$ Department of Mathematics, University of Medicine \\ and Pharmacy-HCMC, Ho Chi Minh City, Vietnam
}

\begin{abstract}
We develop a method for achieving scalable transmission stabilization and switching of $N$ colliding soliton sequences in optical waveguides with broadband delayed Raman response and narrowband nonlinear gain-loss. We show that dynamics of soliton amplitudes in $N$-sequence transmission is described by a generalized $N$-dimensional predator-prey model. Stability and bifurcation analysis for the predator-prey model are used to obtain simple conditions on the physical parameters for robust transmission stabilization as well as on-off and off-on switching of $M$ out of $N$ soliton sequences. Numerical simulations for single-waveguide transmission with a system of $N$ coupled nonlinear Schrödinger equations with $2 \leq N \leq 4$ show excellent agreement with the predator-prey model's predictions and stable propagation over significantly larger distances compared with other broadband nonlinear single-waveguide systems. Moreover, stable on-off and off-on switching of multiple soliton sequences and stable multiple transmission switching events are demonstrated by the simulations. We discuss the reasons for the robustness and scalability of transmission stabilization and switching in waveguides with broadband delayed Raman response and narrowband nonlinear gain-loss, and explain their advantages compared with other broadband nonlinear waveguides.
\end{abstract}

PACS numbers: 42.65.Tg, 05.45.Yv, 42.65.Dr, 42.65.Sf 


\section{INTRODUCTION}

The rates of information transmission through broadband optical waveguide links can be significantly increased by transmitting many pulse sequences through the same waveguide [1 5]. This is achieved by the wavelength-division-multiplexed (WDM) method, where each pulse sequence is characterized by the central frequency of its pulses, and is therefore called a frequency channel [6]. Applications of these WDM or multichannel systems include fiber optics transmission lines [2 5], data transfer between computer processors through silicon waveguides [7 9], and multiwavelength lasers [10 13]. Since pulses from different frequency channels propagate with different group velocities, interchannel pulse collisions are very frequent, and can therefore lead to error generation and severe transmission degradation [1 5 , 14, 15]. On the other hand, the significant collision-induced effects can be used for controlling the propagation, for tuning of optical pulse parameters, such as amplitude, frequency, and phase, and for transmission switching, i.e., the turning on or off of transmission of one or more of the pulse sequences [16 21]. A major advantage of multichannel waveguide systems compared with single-channel systems is that the former can simultaneously handle a large number of pulses using relatively low pulse energies. One of the most important challenges in multichannel transmission concerns the realization of stable scalable control of the transmission, which holds for an arbitrary number of frequency channels. In the current study we address this challenge, by showing that stable scalable transmission control can be achieved in multichannel optical waveguide systems with frequency dependent linear gain-loss, broadband delayed Raman response, and narrowband nonlinear gain-loss.

Interchannel crosstalk, which is the commonly used name for the energy exchange in interchannel collisions, is one of the main processes affecting pulse propagation in broadband waveguide systems. Two important crosstalk-inducing mechanisms are due to broadband delayed Raman response and broadband nonlinear gain-loss. Raman-induced interchannel crosstalk is an important impairment in WDM transmission lines employing silica glass fibers [22 30], but is also beneficially employed for amplification [31, 32]. Interchannel crosstalk due to cubic loss was shown to be a major factor in error generation in multichannel silicon nanowaveguide transmission [33]. Additionally, crosstalk induced by quintic loss can lead to transmission degradation and loss of transmission scalability in multichannel optical waveguides due to the impact of three-pulse interaction on the crosstalk [18, 34]. On the 
other hand, nonlinear gain-loss crosstalk can be used for achieving energy equalization, transmission stabilization, and transmission switching [17-20].

In several earlier studies [16 21], we provided a partial solution to the key problem of achieving stable transmission control in multichannel nonlinear waveguide systems, considering solitons as an example for the optical pulses. Our approach was based on showing that the dynamics of soliton amplitudes in $N$-sequence transmission can be described by Lotka-Volterra (LV) models for $N$ species, where the specific form of the LV model depends on the nature of the dissipative processes in the waveguide. Stability and bifurcation analysis for the steady states of the LV models was used to guide a clever choice of the physical parameters, which in turn leads to transmission stabilization, i.e., the amplitudes of all propagating solitons approach desired predetermined values [16 21]. Furthermore, onoff and off-on transmission switching were demonstrated in two-channel waveguide systems with broadband nonlinear gain-loss [19, 20]. The design of waveguide setups for transmission switching was also guided by stability and bifurcation analysis for the steady states of the LV models [19, 20].

The results of Refs. [16 21] provide the first steps toward employing crosstalk induced by delayed Raman response or by nonlinear gain-loss for transmission control, stabilization, and switching. However, these results are still quite limited, since they do not enable scalable transmission stabilization and switching for $N$ pulse sequences with a general $N$ value in a single optical waveguide. To explain this, we first note that in waveguides with broadband delayed Raman response, such as optical fibers, and in waveguides with broadband cubic loss, such as silicon waveguides, some or all of the soliton sequences propagate in the presence of net linear gain [16, 17, 21]. This leads to transmission destabilization at intermediate distances due to radiative instability and growth of small amplitude waves. As a result, the distances along which stable propagation is observed in these single-waveguide multichannel systems are relatively small even for small values of the Raman and cubic loss coefficients [17, 21]. The radiative instability observed in optical fibers and silicon waveguides can be mitigated by employing waveguides with linear loss, cubic gain, and quintic loss, i.e., waveguides with a Ginzburg-Landau (GL) gain-loss profile [18 20]. However, the latter waveguides suffer from another serious limitation because of the broadband nature of the waveguides nonlinear gain-loss. More specifically, due to the presence of broadband quintic loss, three-pulse interaction gives an important contribution to collision-induced am- 
plitude shifts [18, 34]. The complex nature of three-pulse interaction in generic three-soliton collisions in this case (see Ref. [34]) leads to a major difficulty in extending the LV model for amplitude dynamics from $N=2$ to a general $N$ value in waveguides with broadband nonlinear gain-loss. In the absence of a general $N$-dimensional LV model, it is unclear how to design setups for stable transmission stabilization and switching in $N$-sequence systems with $N>2$. For this reason, transmission stabilization and switching in waveguides with broadband nonlinear gain-loss were so far limited to two-sequence systems [18-20].

In view of the limitations of the waveguides studied in Refs. [16 21], it is important to look for new routes for realizing scalable transmission stabilization and switching, which work for $N$-sequence transmission with a general $N$ value. In the current paper we take on this task, by studying propagation of $N$ soliton sequences in nonlinear waveguides with frequency dependent linear gain-loss, broadband delayed Raman response, and narrowband nonlinear gain-loss. Due to the narrowband nature of the nonlinear gain-loss, it affects only single-pulse propagation and intrasequence interaction, but does not affect intersequence soliton collisions. We show that the combination of Raman-induced amplitude shifts in intersequence soliton collisions and single-pulse amplitude shifts due to gain-loss with properly chosen physical parameter values can be used to realize robust scalable trasmission stabilization and switching. For this purpose, we first obtain the generalized $N$-dimensional predator-prey model for amplitude dynamics in an $N$-sequence system. We then use stability and bifurcation analysis for the predator-prey model to obtain simple conditions on the values of the physical parameters, which lead to robust transmission stabilization as well as on-off and off-on switching of $M$ out of $N$ soliton sequences. The validity of the predator-prey model's predictions is checked by carrying out numerical simulations with the full propagation model, which consists of a system of $N$ perturbed coupled nonlinear Schrödinger (NLS) equations. Our numerical simulations with $2 \leq N \leq 4$ soliton sequences show excellent agreement with the predator-prey model's predictions and stable propagation over significantly larger distances compared with other broadband nonlinear single-waveguide systems. Moreover, stable on-off and off-on switching of multiple soliton sequences and stable multiple transmission switching events are demonstrated by the simulations. We discuss the reasons for the robustness and scalability of transmission stabilization and switching in waveguides with broadband delayed Raman response and narrowband nonlinear gain-loss, and explain their advantages compared with other broadband nonlinear waveguides. 
The rest of the paper is organized as follows. In Section [II, we present the coupled-NLS model for propagation of $N$ pulse sequences through waveguides with frequency dependent linear gain-loss, broadband delayed Raman response, and narrowband nonlinear gain-loss. In addition, we present the corresponding generalized $N$-dimensional predator-prey model for amplitude dynamics. In Section III, we carry out stability and bifurcation analysis for the steady states of the predator-prey model, and use the results to derive conditions on the values of the physical parameters for achieving scalable transmission stabilization and switching. In Section IV] we present the results of numerical simulations with the coupledNLS model for transmission stabilization, single switching events, and multiple transmission switching. We also analyze these results in comparison with the predictions of the predatorprey model. In Section $\nabla$, we discuss the underlying reasons for the robustness and scalability of transmission stabilization and switching in waveguides with broadband delayed Raman response and narrowband nonlinear gain-loss. Section VI is reserved for conclusions.

\section{COUPLED-NLS AND PREDATOR-PREY MODELS}

\section{A. A coupled-NLS model for pulse propagation}

We consider $N$ sequences of optical pulses, each characterized by pulse frequency, propagating in an optical waveguide in the presence of second-order dispersion, Kerr nonlinearity, frequency dependent linear gain-loss, broadband delayed Raman response, and narrowband nonlinear gain-loss. We assume that the net linear gain-loss is the difference between am-

plifier gain and waveguide loss and that the frequency differences between all sequences are much larger than the spectral width of the pulses. Under these assumptions, the propagation is described by the following system of $N$ perturbed coupled-NLS equations:

$$
\begin{aligned}
& i \partial_{z} \psi_{j}+\partial_{t}^{2} \psi_{j}+2\left|\psi_{j}\right|^{2} \psi_{j}+4 \sum_{k=1}^{N}\left(1-\delta_{j k}\right)\left|\psi_{k}\right|^{2} \psi_{j}= \\
& i g_{j} \psi_{j} / 2+i L\left(\left|\psi_{j}\right|^{2}\right) \psi_{j}-\epsilon_{R} \psi_{j} \partial_{t}\left|\psi_{j}\right|^{2}-\epsilon_{R} \sum_{k=1}^{N}\left(1-\delta_{j k}\right)\left[\psi_{j} \partial_{t}\left|\psi_{k}\right|^{2}+\psi_{k} \partial_{t}\left(\psi_{j} \psi_{k}^{*}\right)\right],
\end{aligned}
$$

where $\psi_{j}$ is proportional to the envelope of the electric field of the $j$ th sequence, $1 \leq j \leq N$, $z$ is propagation distance, and $t$ is time. In Eq. (1), $g_{j}$ is the linear gain-loss coefficient for the $j$ th sequence, $\epsilon_{R}$ is the Raman coefficient, and $L\left(\left|\psi_{j}\right|^{2}\right)$ is a polynomial of $\left|\psi_{j}\right|^{2}$, 
describing the waveguide's nonlinear gain-loss profile. The values of the $g_{j}$ coefficients are determined by the $N$-dimensional predator-prey model for amplitude dynamics, by looking for steady-state transmission with equal amplitudes for all sequences. The second term on the left-hand side of Eq. (11) is due to second-order dispersion, while the third and fourth terms represent intrasequence and intersequence interaction due to Kerr nonlinearity. The first term on the right-hand side of Eq. (1) is due to linear gain-loss, the second corresponds to intrasequence interaction due to nonlinear gain-loss, the third describes Raman-induced intrasequence interaction, while the fourth and fifth describe Raman-induced intersequence interaction. Since we consider waveguides with broadband delayed Raman response and narrowband nonlinear gain-loss, Raman-induced intersequence interaction is taken into account, while intersequence interaction due to nonlinear gain-loss is neglected. The polynomial $L$ in Eq. (11) can be quite general. In the current paper, we consider two central examples for waveguide systems with nonlinear gain-loss: (1) waveguides with a GL gain-loss profile, (2) waveguides with linear gain-loss and cubic loss. The expression for $L\left(\left|\psi_{j}\right|^{2}\right)$ for waveguides with a GL gain-loss profile is

$$
L_{1}\left(\left|\psi_{j}\right|^{2}\right)=\epsilon_{3}^{(1)}\left|\psi_{j}\right|^{2}-\epsilon_{5}\left|\psi_{j}\right|^{4}
$$

where $\epsilon_{3}^{(1)}$ and $\epsilon_{5}$ are the cubic gain and quintic loss coefficients. The expression for $L\left(\left|\psi_{j}\right|^{2}\right)$ for waveguides with linear gain-loss and cubic loss is

$$
L_{2}\left(\left|\psi_{j}\right|^{2}\right)=-\epsilon_{3}^{(2)}\left|\psi_{j}\right|^{2}
$$

where $\epsilon_{3}^{(2)}$ is the cubic loss coefficient. We emphasize, however, that our approach can be employed to treat a general form of the polynomial $L$. Note that since some of the perturbation terms in the propagation model (1) are nonlinear gain or loss terms, the model can also be regarded as a coupled system of GL equations.

The dimensional and dimensionless physical quantities are related by the standard scaling laws for NLS solitons [1]. Exactly the same scaling relations were used in our previous works on soliton propagation in broadband nonlinear waveguide systems [17-21]. In these scaling relations, the dimensionless distance $z$ in Eq. (1) is $z=X /\left(2 L_{D}\right)$, where $X$ is the dimensional distance, $L_{D}=\tau_{0}^{2} /\left|\tilde{\beta}_{2}\right|$ is the dimensional dispersion length, $\tau_{0}$ is the soliton width, and $\tilde{\beta}_{2}$ is the second-order dispersion coefficient. The dimensionless retarded time is $t=\tau / \tau_{0}$, where $\tau$ is the retarded time. The solitons spectral width is $\nu_{0}=1 /\left(\pi^{2} \tau_{0}\right)$ 
and the frequency difference between adjacent channels is $\Delta \nu=\left(\pi \Delta \beta \nu_{0}\right) / 2 . \psi_{j}=E_{j} / \sqrt{P_{0}}$, where $E_{j}$ is proportional to the electric field of the $j$ th pulse sequence and $P_{0}$ is the peak power. The dimensionless second order dispersion coefficient is $d=-1=\tilde{\beta}_{2} /\left(\gamma P_{0} \tau_{0}^{2}\right)$, where $\gamma$ is the Kerr nonlinearity coefficient. The dimensional linear gain-loss coefficient for the $j$ th sequence $\rho_{1 j}^{(l)}$ is related to the dimensionless coefficient via $g_{j}^{(l)}=2 \rho_{1 j}^{(l)} /\left(\gamma P_{0}\right)$. The coefficients $\epsilon_{3}^{(1)}, \epsilon_{3}^{(2)}$, and $\epsilon_{5}$ are related to the dimensional cubic gain $\rho_{3}^{(1)}$, cubic loss $\rho_{3}^{(2)}$, and quintic loss $\rho_{5}$, by $\epsilon_{3}^{(1)}=2 \rho_{3}^{(1)} / \gamma, \epsilon_{3}^{(2)}=2 \rho_{3}^{(2)} / \gamma$, and $\epsilon_{5}=2 \rho_{5} P_{0} / \gamma$, respectively [20]. The dimensionless Raman coefficient is $\epsilon_{R}=2 \tau_{R} / \tau_{0}$, where $\tau_{R}$ is a dimensional time constant, characterizing the waveguide's delayed Raman response [1, 43]. The time constant $\tau_{R}$ can be determined from the slope of the Raman gain curve of the waveguide [1, 43].

We note that for waveguides with linear gain-loss and cubic loss, some or all of the pulse sequences propagate in the presence of net linear gain. This leads to transmission destabilization due to radiation emission. The radiative instability can be partially mitigated by employing frequency dependent linear gain-loss $g(\omega, z)$. In this case, the first term on the right hand side of Eq. (11) is replaced by $i \mathcal{F}^{-1}\left(g(\omega, z) \hat{\psi}_{j}\right) / 2$, where $\hat{\psi}$ is the Fourier transform of $\psi$ with respect to time, and $\mathcal{F}^{-1}$ stands for the inverse Fourier transform. The form of $g(\omega, z)$ is chosen such that existence of steady-state transmission with equal amplitudes for all sequences is retained, while radiation emission effects are minimized. More specifically, $g(\omega, z)$ is equal to a value $g_{j}$, required to balance amplitude shifts due to nonlinear gain-loss and Raman crosstalk, inside a frequency interval of width $W$ centered about the frequency of the $j$ th-channel solitons at distance $z, \beta_{j}(z)$, and is equal to a negative value $g_{L}$ elsewhere [35]. Thus, $g(\omega, z)$ is given by:

$$
g(\omega, z)= \begin{cases}g_{j} & \text { if } \beta_{j}(z)-W / 2<\omega \leq \beta_{j}(z)+W / 2 \text { for } 1 \leq j \leq N \\ g_{L} \text { elsewhere }\end{cases}
$$

where $g_{L}<0$. The width $W$ in Eq. (44) satisfies $1<W \leq \Delta \beta$, where $\Delta \beta=\beta_{j+1}(0)-\beta_{j}(0)$ for $1 \leq j \leq N-1$. The values of the $g_{j}$ coefficients are determined by the generalized predator-prey model for collision-induced amplitude dynamics, such that amplitude shifts due to Raman crosstalk and nonlinear gain-loss are compensated for by the linear gain-loss. The values of $g_{L}$ and $W$ are determined by carrying out numerical simulations with Eqs. (1), (3), and (44), while looking for the set, which yields the longest stable propagation distance 


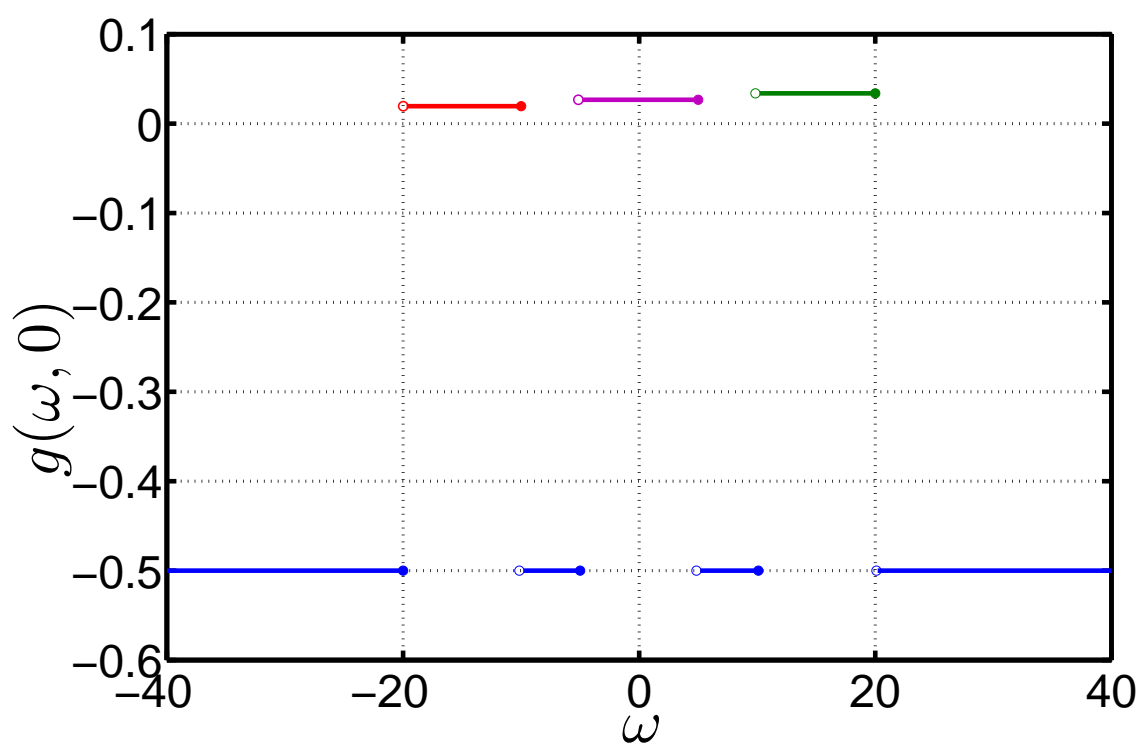

FIG. 1: An example for the frequency dependent linear gain-loss function $g(\omega, z)$ of Eq. (44) at $z=0$ in a three-channel system.

[35]. Figure 1 shows a typical example for the frequency dependent linear gain-loss function $g(\omega, z)$ at $z=0$ for a three-channel system with $g_{1}=0.0195, g_{2}=0.0267, g_{3}=0.0339$, $g_{L}=-0.5, \beta_{1}(0)=-15, \beta_{2}(0)=0, \beta_{3}(0)=15$, and $W=10$. These parameter values are used in the numerical simulations, whose results are shown in Fig. 7.

The optical pulses in the $j$ th sequence are fundamental solitons of the unperturbed NLS equation with central frequency $\beta_{j}$. The envelopes of these solitons are given by $\psi_{s j}(t, z)=$ $\eta_{j} \exp \left(i \chi_{j}\right) \operatorname{sech}\left(x_{j}\right)$, where $x_{j}=\eta_{j}\left(t-y_{j}-2 \beta_{j} z\right), \chi_{j}=\alpha_{j}+\beta_{j}\left(t-y_{j}\right)+\left(\eta_{j}^{2}-\beta_{j}^{2}\right) z$, and $\eta_{j}, y_{j}$, and $\alpha_{j}$ are the soliton amplitude, position, and phase. Due to the large frequency differences between the pulse sequences, the solitons undergo a large number of fast intersequence collisions. The energy exchange in the collisions, induced by broadband delayed Raman response, can lead to significant amplitude shifts and to transmission degradation. On the other hand, the combination of Raman-induced amplitude shifts in intersequence collisions and single-pulse amplitude shifts due to frequency dependent linear gain-loss and narrowband nonlinear gain-loss with properly chosen coefficients can be used to realize robust scalable transmission stabilization and switching. In the current paper, we demonstrate that such stable scalable transmission control can indeed be achieved, even with the simple nonlinear gain-loss profiles (2) and (3) 


\section{B. A generalized $N$-dimensional predator-prey model for amplitude dynamics}

The design of waveguide setups for transmission stabilization and switching is based on the derivation of LV models for dynamics of soliton amplitudes. For this purpose, we consider propagation of $N$ soliton sequences in a waveguide loop, and assume that the frequency spacing $\Delta \beta$ between the sequences is a large constant, i.e., $\Delta \beta=\left|\beta_{j+1}(z)-\beta_{j}(z)\right| \gg 1$ for $1 \leq j \leq N-1$. Similar to Refs. [16, 17], we can show that amplitude dynamics of the $N$ sequences is approximately described by a generalized $N$-dimensional predator-prey model. The derivation of the predator-prey model is based on the following assumptions. (1) The temporal separation $T$ between adjacent solitons in each sequence satisfies: $T \gg$ 1. In addition, the amplitudes are equal for all solitons from the same sequence, but are not necessarily equal for solitons from different sequences. This setup corresponds, for example, to phase-shift-keyed soliton transmission. (2) As $T \gg 1$, intrasequence interaction is exponentially small and is neglected. (3) Delayed Raman response and gain-loss are assumed to be weak perturbations. As a result, high-order effects due to radiation emission are neglected, in accordance with single-collision analysis.

Since the pulse sequences are periodic, the amplitudes of all solitons in a given sequence undergo the same dynamics. Taking into account collision-induced amplitude shifts due to broadband delayed Raman response and single-pulse amplitude changes induced by gain and loss, we obtain the following equation for amplitude dynamics of the $j$ th-sequence solitons (see Refs. [16, 17] for similar derivations):

$$
\frac{d \eta_{j}}{d z}=\eta_{j}\left[g_{j}+F\left(\eta_{j}\right)+C \sum_{k=1}^{N}(k-j) f(|j-k|) \eta_{k}\right]
$$

where $1 \leq j \leq N$, and $C=4 \epsilon_{R} \Delta \beta / T$. The function $F\left(\eta_{j}\right)$ on the right hand side of Eq. (5) is a polynomial in $\eta_{j}$, whose form is determined by the form of $L\left(\left|\psi_{j}\right|^{2}\right)$. For $L_{1}$ and $L_{2}$ given by Eqs. (21) and (3), we obtain $F_{1}\left(\eta_{j}\right)=4 \epsilon_{3}^{(1)} \eta_{j}^{2} / 3-16 \epsilon_{5} \eta_{j}^{4} / 15$ and $F_{2}\left(\eta_{j}\right)=-4 \epsilon_{3}^{(2)} \eta_{j}^{2} / 3$, respectively. The coefficients $f(|j-k|)$ on the right hand side of Eq. (5), which describe the strength of Raman interaction between $j$ th- and $k$ th-sequence solitons, are determined by the frequency dependence of the Raman gain. For the widely used triangular approximation for the Raman gain curve [1, 22], in which the gain is a piecewise linear function of the frequency, $f(|j-k|)=1$ for $1 \leq j \leq N$ and $1 \leq k \leq N$ [16].

In order to demonstrate stable scalable control of soliton propagation, we look for an 
equilibrium state of the system (5) in the form $\eta_{j}^{(e q)}=\eta>0$ for $1 \leq j \leq N$. Such equilibrium state corresponds to steady-state transmission with equal amplitudes for all sequences. This requirement leads to:

$$
g_{j}=-F(\eta)-C \eta \sum_{k=1}^{N}(k-j) f(|j-k|) .
$$

Consequently, Eq. (5) takes the form

$$
\frac{d \eta_{j}}{d z}=\eta_{j}\left[F\left(\eta_{j}\right)-F(\eta)+C \sum_{k=1}^{N}(k-j) f(|j-k|)\left(\eta_{k}-\eta\right)\right]
$$

which is a generalized predator-prey model for $N$ species [36, 37]. Notice that $(\eta, \ldots, \eta)$ and $(0, \ldots, 0)$ are equilibrium states of the model for any positive values of $\epsilon_{3}^{(1)}, \epsilon_{3}^{(2)}, \epsilon_{5}, \eta$, and $C$.

We point out that the derivation of an $N$-dimensional predator-prey model with a general $N$ value is enabled by the narrow bandwidth of the waveguide's nonlinear gain-loss. Indeed, due to this property, the gain-loss does not contribute to amplitude shifts in interchannel collisions, and therefore, three-pulse interaction can be ignored. This makes the extension of the predator-prey model from $N=2$ to a general $N$ value straightforward. As a result, extending waveguide setup design from $N=2$ to a general $N$ value for waveguides with broadband delayed Raman response and narrowband nonlinear gain-loss is also straightforward. This situation is very different from the one encountered for waveguides with broadband nonlinear gain-loss. In the latter case, interchannel collisions are strongly affected by the nonlinear gain-loss, and three-pulse interaction gives an important contribution to the collision-induced amplitude shift [18, 34]. Due to the complex nature of three-pulse interaction in generic three-soliton collisions in waveguides with broadband nonlinear gain or loss (see Ref. [34]), it is very difficult to extend the LV model for amplitude dynamics from $N=2$ to a generic $N$ value for these waveguides. In the absence of an $N$-dimensional $\mathrm{LV}$ model, it is unclear how to design setups for stable transmission stabilization and switching in $N$-sequence systems with $N>2$. As a result, transmission stabilization and switching in waveguides with broadband nonlinear gain-loss have been so far limited to two-sequence systems $[18-20]$. 


\section{STABILITY ANALYSIS FOR THE PREDATOR-PREY MODEL (7)}

\section{A. Introduction}

Transmission stabilization and switching are guided by stability analysis of the equilibrium states of the predator-prey model (7). In particular, in transmission stabilization, we require that the equilibrium state $(\eta, \ldots, \eta)$ is asymptotically stable, so that soliton amplitude values tend to $\eta$ with increasing propagation distance for all sequences. Furthermore, transmission switching is based on bifurcations of the equilibrium state $(\eta, \ldots, \eta)$. To explain this, we denote by $\eta_{t h}$ the value of the decision level, distinguishing between on and off transmission states, and consider transmission switching of $M$ sequences, for example. In off-on switching of $M$ sequences, the values of one or more of the physical parameters are changed at the switching distance $z_{s}$, such that $(\eta, \ldots, \eta)$ turns from unstable to asymptotically stable. As a result, before switching, soliton amplitudes tend to values smaller than $\eta_{t h}$ in $M$ sequences and to values larger than $\eta_{t h}$ in $N-M$ sequences, while after the switching, soliton amplitudes in all $N$ sequences tend to $\eta$, where $\eta>\eta_{t h}$. This means that transmission of $M$ sequences is turned on at $z>z_{s}$. On-off switching of $M$ sequences is realized by changing the physical parameters at $z=z_{s}$, such that $(\eta, \ldots, \eta)$ turns from asymptotically stable to unstable, while another equilibrium state with $M$ components smaller than $\eta_{t h}$ is asymptotically stable. Therefore, before switching, soliton amplitudes in all $N$ sequences tend to $\eta$, where $\eta>\eta_{t h}$, while after the switching, soliton amplitudes tend to values smaller than $\eta_{t h}$ in $M$ sequences and to values larger than $\eta_{t h}$ in $N-M$ sequences. Thus, transmission of $M$ sequences is turned off at $z>z_{s}$ in this case. In both transmission stabilization and switching we require that the equilibrium state at the origin is asymptotically stable. This requirement is necessary in order to suppress radiative instability due to growth of small amplitude waves [18-20].

The setups of transmission switching that we develop and study in the current paper are different from the single-pulse switching setups that are commonly considered in nonlinear optics (see, e.g., Ref. [1] for a description of the latter setups). We therefore point out the main differences between the two approaches to switching. First, in the common approach, the amplitude value in the off state is close to zero. In contrast, in our approach, the amplitude value in the off state only needs to be smaller than $\eta_{t h}$, although the possibility to extend 
the switching to very small amplitude values does increase switching robustness. Second, in the common approach, the switching is based on a single collision or on a small number of collisions, and as a result, it often requires high-energy pulses for its implementation. In contrast, in our approach, the switching occurs as a result of the cumulative amplitude shift in a large number of fast interchannel collisions. Therefore, in this case pulse energies need not be high. Third and most important, in the common approach, the switching is carried out on a single pulse or on a few pulses. In contrast, in our approach, the switching is carried out on all pulses in the waveguide loop (or within a given waveguide span). As a result, the switching can be implemented with an arbitrary number of pulses. Because of this property, we can refer to transmission switching in our approach as channel switching. Since channel switching is carried out for all pulses inside the waveguide loop (or inside a given waveguide span), it can be much faster than conventional single-pulse switching. More specifically, channel switching can be faster by a factor of $M \times K$ compared with single-pulse switching, where $M$ is the number of channels, whose transmission is switched, and $K$ is the number of pulses per channel in the waveguide loop. For example, in a 100-channel system with $10^{4}$ pulses per channel, channel switching can be faster by a factor of $10^{6}$ compared with single-pulse switching.

Our channel switching approach can be used in any application, in which the same "processing" of all pulses within the same channel is required, where here processing can mean amplification, filtering, routing, signal processing, etc. A simple and widely known example for channel switching is provided by transmission recovery, i.e., the amplification of a sequence of pulses from small amplitudes values below $\eta_{t h}$ to a desired final value above it. However, our channel switching approach can actually be used in a much more general and sophisticated manner. More specifically, let $p_{j}$ represent the transmission state of the $j$ th channel, i.e., $p_{j}=0$ if the $j$ th channel is off and $p_{j}=1$ if the $j$ th channel is on. Then, the $N$-component vector $\left(p_{1}, \ldots, p_{j}, \ldots, p_{N}\right)$, where $1 \leq j \leq N$, represents the transmission state of the entire $N$-channel system. One can then use this $N$-component vector to encode information about the processing to be carried out on different channels in the next "processing station" in the transmission line. After this processing has been carried out, the transmission state of the system can be switched to a new state, $\left(q_{1}, \ldots, q_{j}, \ldots, q_{N}\right)$, which represents the type of processing to be carried out in the next processing station. Note that the channel switching approach is especially suitable for phase-shift-keyed transmission. Indeed, in this 
case, the phase is used for encoding the information, and therefore, no information is lost by operating with amplitude values smaller than $\eta_{t h}[38]$.

\section{B. Stability analysis for transmission stabilization and off-on switching}

Let us obtain the conditions on the values of the physical parameters for transmission stabilization and off-on switching. As explained above, in this case we require that both $(\eta, \ldots, \eta)$ and the origin are asymptotically stable equilibrium states of the predator-prey model (7).

We first analyze stability of the equilibrium state $(\eta, \ldots, \eta)$ in a waveguide with a narrowband GL gain-loss profile, where $F\left(\eta_{j}\right)=F_{1}\left(\eta_{j}\right)$. For this purpose, we show that

$$
V_{L}(\boldsymbol{\eta})=\sum_{j=1}^{N}\left[\eta_{j}-\eta+\eta \ln \left(\eta / \eta_{j}\right)\right]
$$

where $\boldsymbol{\eta}=\left(\eta_{1}, \ldots, \eta_{j}, \ldots, \eta_{N}\right)$, is a Lyapunov function for Eq. (77) [39]. Indeed, we observe that $V_{L}(\boldsymbol{\eta}) \geq 0$ for any $\boldsymbol{\eta}$ with $\eta_{j}>0$ for $1 \leq j \leq N$, where equality holds only at the equilibrium point. Furthermore, the derivative of $V_{L}$ along trajectories of Eq. (7) satisfies:

$$
d V_{L} / d z=-\left(16 \epsilon_{5} / 15\right) \sum_{j=1}^{N}\left(\eta_{j}+\eta\right)\left(\eta_{j}-\eta\right)^{2} \times\left(\eta_{j}^{2}+\eta^{2}-5 \kappa / 4\right),
$$

where $\kappa=\epsilon_{3}^{(1)} / \epsilon_{5}$ and $\epsilon_{5} \neq 0$. For asymptotic stability, we require $d V_{L} / d z<0$. This condition is satisfied in a domain containing $(\eta, \ldots, \eta)$ if $0<\kappa<8 \eta^{2} / 5$. Thus, $V_{L}(\boldsymbol{\eta})$ is a Lyapunov function for Eq. (7), and the equilibrium point $(\eta, \ldots, \eta)$ is asymptotically stable, if $0<\kappa<8 \eta^{2} / 5$ [40]. When $0<\kappa \leq 4 \eta^{2} / 5,(\eta, \ldots, \eta)$ is globally asymptotically stable, since in this case, $d V_{L} / d z<0$ for any initial condition with nonzero amplitude values. When $4 \eta^{2} / 5<\kappa<8 \eta^{2} / 5, d V_{L} / d z<0$ for amplitude values satisfying $\eta_{j}>\left(5 \kappa / 4-\eta^{2}\right)^{1 / 2}$ for $1 \leq j \leq N$. Thus, in this case the basin of attraction of $(\eta, \ldots, \eta)$ can be estimated by $\left(\left(5 \kappa / 4-\eta^{2}\right)^{1 / 2}, \infty\right)$ for $1 \leq j \leq N$. For instability, we require $d V_{L} / d z>0$ along trajectories of (7). This inequality is satisfied in a domain containing $(\eta, \ldots, \eta)$ if $\kappa>8 \eta^{2} / 5$. Therefore, $(\eta, \ldots, \eta)$ is unstable for $\kappa>8 \eta^{2} / 5[40]$.

Consider now the stability properties of the origin for $F\left(\eta_{j}\right)=F_{1}\left(\eta_{j}\right)$. Linear stability analysis shows that $(0, \ldots, 0)$ is asymptotically stable (a stable node) when $g_{j}<0$ for $1 \leq j \leq N$, i.e., when all pulse sequences propagate in the presence of net linear loss. To 
slightly simplify the discussion, we now employ the widely accepted triangular approximation for the Raman gain curve [1, 22]. In this case, $f(|j-k|)=1$ for $1 \leq j \leq N$ and $1 \leq k \leq N$ [16], and therefore the net linear gain-loss coefficients take the form

$$
g_{j}=-F_{1}(\eta)-C N(N+1) \eta / 2+C N \eta j
$$

Since $g_{j}$ is increasing with increasing $j$, it is sufficient to require $g_{N}<0$. Substituting Eq. (10) into this inequality, we find that the origin is asymptotically stable, provided that

$$
\kappa>4 \eta^{2} / 5+3 C N(N-1) /\left(8 \epsilon_{5} \eta\right)
$$

The same simple condition is obtained by showing that $V_{L}(\boldsymbol{\eta})=\sum_{j=1}^{N} \eta_{j}^{2}$ is a Lyapunov function for Eq. (17).

Let us discuss the implications of stability analysis for $(\eta, \ldots, \eta)$ and the origin for transmission stabilization and off-on switching. Combining the requirements for asymptotic stability of both $(\eta, \ldots, \eta)$ and the origin, we expect to observe stable long-distance propagation, for which soliton amplitudes in all sequences tend to their steady-state value $\eta$, provided the physical parameters satisfy

$$
4 \eta^{2} / 5+3 C N(N-1) /\left(8 \epsilon_{5} \eta\right)<\kappa<8 \eta^{2} / 5
$$

The same condition is required for realizing stable off-on transmission switching. Using inequality (12), we find that the smallest value of $\epsilon_{5}$, required for transmission stabilization and off-on switching, satisfies the simple condition

$$
\epsilon_{5}>15 C N(N-1) /\left(32 \eta^{3}\right)
$$

As a result, the ratio $\epsilon_{R} / \epsilon_{5}$ should be a small parameter in $N$-sequence transmission with $N \gg 1$. The independence of the stability condition for $(\eta, \ldots, \eta)$ on $N$ and $\epsilon_{R}$ and the simple scaling properties of the stability condition for the origin are essential ingredients in enabling robust scalable transmission stabilization and switching.

Similar stability analysis can be carried out for waveguides with other forms of the nonlinear gain-loss $F\left(\eta_{j}\right)$ [39]. Consider the central example of a waveguide with narrowband cubic loss, where $F\left(\eta_{j}\right)=F_{2}\left(\eta_{j}\right)$. One can show that in this case $V_{L}(\boldsymbol{\eta})$, given by Eq. (8) , is a Lyapunov function for the predator-prey model (7), and that

$$
d V_{L} / d z=-\left(4 \epsilon_{3}^{(2)} / 3\right) \sum_{j=1}^{N}\left(\eta_{j}+\eta\right)\left(\eta_{j}-\eta\right)^{2}<0
$$


for any trajectory with $\eta_{j}>0$ for $1 \leq j \leq N$. Thus, $(\eta, \ldots, \eta)$ is globally asymptotically stable, regardless of the values of $\eta, \epsilon_{R}, \epsilon_{3}^{(2)}$, and $N$. However, linear stability analysis shows that the origin is a saddle in this case, i.e., it is unstable. This instability is related to the fact that in waveguides with cubic loss, soliton sequences with $j$ values satisfying

$j>(N+1) / 2-4 \epsilon_{3}^{(2)} \eta /(3 C N)$ propagate under net linear gain, and are thus subject to radiative instability. The instability of the origin for uniform waveguides with cubic loss makes these waveguides unsuitable for long-distance transmission stabilization. On the other hand, the global stability of $(\eta, \ldots, \eta)$ and its independence on the physical parameters, make waveguide spans with narrowband cubic loss very suitable for realizing robust scalable off-on switching in hybrid waveguides. To demonstrate this, consider a hybrid waveguide consisting of spans with linear gain-loss and cubic loss $\left[F\left(\eta_{j}\right)=F_{2}\left(\eta_{j}\right)\right]$ and spans with a GL gain-loss profile $\left[F\left(\eta_{j}\right)=F_{1}\left(\eta_{j}\right)\right]$. In this case, the global stability of $(\eta, \ldots, \eta)$ for spans with linear gain-loss and cubic loss can be used to bring amplitude values close to $\eta$ from small initial amplitude values, while the local stability of the origin for spans with a GL gain-loss profile can be employed to stabilize the propagation against radiation emission.

\section{Stability analysis for on-off switching}

We now describe stability analysis for on-off switching in waveguides with a GL gain-loss profile, considering the general case of switching off of $M$ out of $N$ soliton sequences. As explained in Subsection 3.1, in switching off of $M$ sequences, we require that $(\eta, \ldots, \eta)$ is unstable, the origin is asymptotically stable, and another equilibrium state with $M$ components smaller than $\eta_{t h}$ is also asymptotically stable. The requirement for instability of $(\eta, \ldots, \eta)$ and asymptotic stability of the origin leads to the following condition on the physical parameter values:

$$
\kappa>\max \left\{8 \eta^{2} / 5,4 \eta^{2} / 5+3 C N(N-1) /\left(8 \epsilon_{5} \eta\right)\right\} .
$$

In order to obtain guiding rules for choosing the on-off transmission switching setups, it is useful to consider first the case of switching off of N-1 out of N sequences. Suppose that we switch off the sequences $1 \leq k \leq j-1$ and $j+1 \leq k \leq N$. To realize such switching, we require that $\left(0, \ldots, 0, \eta_{s j}, 0, \ldots, 0\right)$ is a stable equilibrium point of Eq. (7). The value of $\eta_{s j}$ 
is determined by the equation

$$
\eta_{s j}^{4}-5 \kappa \eta_{s j}^{2} / 4-15 g_{j} /\left(16 \epsilon_{5}\right)=0
$$

Since the origin is a stable equilibrium point, transmission switching of $N-1$ sequences can be realized by requiring that Eq. (16) has two distinct roots on the positive half of the $\eta_{j}$-axis (the largest of which corresponds to $\eta_{s j}$ ). This requirement is satisfied, provided [41]:

$$
\epsilon_{5}>12\left|g_{j}\right| /\left(5 \kappa^{2}\right) \text {. }
$$

Assuming that $g_{1}<g_{2}<\cdots<g_{N}<0$, we see that the switching off of the $N-1$ low-frequency sequences $1 \leq j \leq N-1$ is the least restrictive, since it can be realized with smaller $\epsilon_{5}$ values. For this reason, we choose to adopt the switching setup, in which sequences $1 \leq j \leq N-1$ are switched off. Employing inequality (17) and the triangularapproximation-based expression (10) for $j=N$, we find that Eq. (16) has two distinct roots on the positive half of the $\eta_{N}$-axis, provided that

$$
\kappa>(8 \eta / 5)\left[5 \kappa / 4-\eta^{2}-15 C N(N-1) /\left(32 \epsilon_{5} \eta\right)\right]^{1 / 2} .
$$

Therefore, the switching off of sequences $1 \leq j \leq N-1$ can be realized when conditions (15) and (18) are satisfied [42].

We now turn to discuss the general case, where transmission of $M$ out of $N$ sequences is switched off. Based on the discussion in the previous paragraph, one might expect that switching off of $M$ sequences can be most conveniently realized by turning off transmission of the low-frequency sequences, $1 \leq j \leq M$. This expectation is confirmed by numerical solution of the predator-prey model (17) and the coupled-NLS model (11). For this reason, we choose to employ switching off of $M$ sequences, in which transmission in the $M$ lowest frequency channels is turned off. Thus, we require that $\left(0, \ldots, 0, \eta_{s(M+1)}, \ldots, \eta_{s N}\right)$ is an asymptotically stable equilibrium point of Eq. (17). The values of $\eta_{s(M+1)}, \ldots, \eta_{s N}$ are determined by the following system of equations

$$
\eta_{s j}^{4}-5 \kappa \eta_{s j}^{2} / 4-15 g_{j} /\left(16 \epsilon_{5}\right)-15 C /\left(16 \epsilon_{5}\right) \sum_{k=M+1}^{N}(k-j) f(|j-k|) \eta_{s k}=0
$$

where $M+1 \leq j \leq N$. Employing the triangular approximation for the Raman gain curve and using Eq. (10), we can rewrite the system as:

$$
\begin{aligned}
& \eta_{s j}^{4}-5 \kappa \eta_{s j}^{2} / 4-15 C /\left(16 \epsilon_{5}\right) \sum_{k=M+1}^{N}(k-j) \eta_{s k}-\eta^{4}+5 \kappa \eta^{2} / 4 \\
& +15 C N[(N+1) / 2-j] \eta /\left(16 \epsilon_{5}\right)=0 .
\end{aligned}
$$


Stability of $\left(0, \ldots, 0, \eta_{s(M+1)}, \ldots, \eta_{s N}\right)$ is determined by calculating the eigenvalues of the Jacobian matrix $\mathcal{J}$ at this point. The calculation yields $\mathcal{J}_{j k}=0$ for $1 \leq j \leq M$ and $j \neq k$,

$$
\begin{gathered}
\mathcal{J}_{j j}=-4 \epsilon_{3}^{(1)} \eta^{2} / 3+16 \epsilon_{5} \eta^{4} / 15-C\left[N(N+1) \eta / 2-\sum_{k=M+1}^{N} k \eta_{s k}\right] \\
+C\left[N \eta-\sum_{k=M+1}^{N} \eta_{s k}\right] j \quad \text { for } 1 \leq j \leq M, \\
\mathcal{J}_{j k}=C(k-j) \eta_{s j} \text { for } M+1 \leq j \leq N \text { and } j \neq k,
\end{gathered}
$$

and

$$
\mathcal{J}_{j j}=g_{j}+4 \epsilon_{3}^{(1)} \eta_{s j}^{2}-16 \epsilon_{5} \eta_{s j}^{4} / 3+C \sum_{k=M+1}^{N}(k-j) \eta_{s k} \text { for } M+1 \leq j \leq N .
$$

Note that the Raman triangular approximation was used to slightly simplify the form of Eqs. (21) $-(\underline{23})$. Since $\mathcal{J}_{j k}=0$ for $1 \leq j \leq M$ and $j \neq k$, the first $M$ eigenvalues of the Jacobian matrix are $\lambda_{j}=\mathcal{J}_{j j}$, where the $\mathcal{J}_{j j}$ coefficients are given by Eq. (21). Furthermore, since $\mathcal{J}_{j j}$ is either monotonically increasing or monotonically decreasing with increasing $j$, to establish stability, it is sufficient to check that either $\mathcal{J}_{M M}<0$ or $\mathcal{J}_{11}<0$. To find the other $N-M$ eigenvalues of the Jacobian matrix, one needs to calculate the determinant of the $(N-M) \times(N-M)$ matrix, whose elements are $\mathcal{J}_{j k}$, where $M+1 \leq j, k \leq N$. The latter calculation can also be significantly simplified by noting that for $M+1 \leq j \leq N$, the diagonal elements are of order $\epsilon_{5}$, while the off-diagonal elements are of order $N \epsilon_{R}$ at most. Thus, the leading term in the expression for the determinant is of order $\epsilon_{5}^{N-M}$. The next term in the expansion is the sum of $N-M$ terms, each of which is of order $N^{2} \epsilon_{R}^{2} \epsilon_{5}^{N-M-2}$ at most. Therefore, the next term in the expansion of the determinant is of order $(N-M) N^{2} \epsilon_{R}^{2} \epsilon_{5}^{N-M-2}$ at most. Comparing the first and second terms, we see that the correction term can be neglected, provided that $\epsilon_{5} \gg N^{3 / 2} \epsilon_{R}$. We observe that the last condition is automatically satisfied by our on-off transmission switching setup for $N \gg 1$, since stability of the origin requires $\epsilon_{5}>N^{2} \epsilon_{R} \gg N^{3 / 2} \epsilon_{R}$ [see inequality (15)]. It follows that the other $N-M$ eigenvalues of the Jacobian matrix are well approximated by the diagonal elements $\mathcal{J}_{j j}$ for $M+1 \leq j \leq N$. Therefore, for $N \gg 1$, stability analysis of $\left(0, \ldots, 0, \eta_{s(M+1)}, \ldots, \eta_{s N}\right)$ only requires the calculation of $N-M+1$ diagonal elements of the Jacobian matrix. 
We point out that the preference for the turning off of transmission of low-frequency sequences in on-off switching is a consequence of the nature of the Raman-induced energy exchange in soliton collisions. Indeed, Raman crosstalk leads to energy transfer from highfrequency solitons to low-frequency ones [26, 43-48]. To compensate for this energy loss or gain, high-frequency sequences should be overamplified while low-frequency sequences should be underamplified compared to mid-frequency sequences [16, 21]. As a result, the magnitude of the net linear loss is largest for the low-frequency sequences, and therefore, on-off switching is easiest to realize for these sequences. It follows that the presence of broadband delayed Raman response introduces a preference for turning off the transmission of the low-frequency sequences, and by this, enables systematic scalable on-off switching in $N$-sequence systems.

\section{NUMERICAL SIMULATIONS WITH THE COUPLED-NLS MODEL}

The predator-prey model (7) is based on several simplifying assumptions, which might break down with increasing number of channels or at large propagation distances. In particular, Eq. (7) neglects the effects of pulse distortion, radiation emission, and intrasequence interaction that are incorporated in the full coupled-NLS model (1). These effects can lead to transmission destabilization and to the breakdown of the predator-prey model description [17 21]. In addition, during transmission switching, soliton amplitudes can become small, and as a result, the magnitude of the linear gain-loss term in Eq. (11) might become comparable to the magnitude of the Kerr nonlinearity terms. This can in turn lead to the breakdown of the perturbation theory, which is the basis for the derivation of the predator-prey model. It is therefore essential to test the validity of the predator-prey model's predictions by carrying out numerical simulations with the full coupled-NLS model (11).

The coupled-NLS system (1) is numerically integrated using the split-step method with periodic boundary conditions [1]. Due to the usage of periodic boundary conditions, the simulations describe pulse propagation in a closed waveguide loop. The initial condition for the simulations consists of $N$ periodic sequences of $2 K$ solitons with amplitudes $\eta_{j}(0)$, frequencies $\beta_{j}(0)$, and zero phases:

$$
\psi_{j}(t, 0)=\sum_{k=-K}^{K-1} \frac{\eta_{j}(0) \exp \left\{i \beta_{j}(0)\left[t-(k+1 / 2) T-\delta_{j}\right]\right\}}{\cosh \left\{\eta_{j}(0)\left[t-(k+1 / 2) T-\delta_{j}\right]\right\}},
$$


where the frequency differences satisfy $\Delta \beta=\beta_{j+1}(0)-\beta_{j}(0) \gg 1$, for $1 \leq j \leq N-1$. The coefficients $\delta_{j}$ represent the initial position shift of the $j$ th sequence solitons relative to pulses located at $(k+1 / 2) T$ for $-K \leq k \leq K-1$. To maximize propagation distance in the presence of delayed Raman response, we use $\delta_{j}=(j-1) T / N$ for $1 \leq j \leq N$. As a concrete example, we present the results of numerical simulations for the following set of physical parameters: $T=15, \Delta \beta=15$, and $K=1$. In addition, we employ the triangular approximation for the Raman gain curve, so that the coefficients $f(|j-k|)$ satisfy $f(|j-k|)=1$ for $1 \leq j, k \leq N$ [16, 21]. We emphasize, however, that similar results are obtained with other choices of the physical parameter values, satisfying the stability conditions discussed in Section III.

We first describe numerical simulations for transmission stabilization in waveguides with broadband delayed Raman response and a narrowband GL gain-loss profile $\left[L\left(\left|\psi_{j}\right|^{2}\right)=\right.$ $\left.L_{1}\left(\left|\psi_{j}\right|^{2}\right)\right]$ for $N=2, N=3$, and $N=4$ sequences. We choose $\eta=1$ so that the desired steady state of the system is $(1, \ldots, 1)$. The Raman coefficient is $\epsilon_{R}=0.0006$, while the quintic loss coefficient is $\epsilon_{5}=0.1$ for $N=2, \epsilon_{5}=0.15$ for and $N=3$, and $\epsilon_{5}=0.25$ for $N=4$. In addition, we choose $\kappa=1.2$ and initial amplitudes satisfying $\eta_{j}(0)>\left(5 \kappa / 4-\eta^{2}\right)^{1 / 2}$ for $1 \leq j \leq N$, so that the initial amplitudes belong to the basin of attraction of $(1, \ldots, 1)$. The numerical simulations with Eqs. (11) and (2) are carried out up to the final distances $z_{f_{1}}=36110, z_{f_{2}}=21320$, and $z_{f_{3}}=5350$, for $N=2, N=3$, and $N=4$, respectively. At these distances, the onset of transmission destabilization due to radiation emission and pulse distortion is observed. The $z$ dependence of soliton amplitudes obtained by the simulations is shown in Figs. 2(a), 2(c), and 2(e) together with the prediction of the predator-prey model (7). Figures 2(b), 2(d), and 2(f) show the amplitude dynamics at short distances. Figures 3(a), 3(c), and 3(e) show the pulse patterns $\left|\psi_{j}(t, z)\right|$ at a distance $z=z_{r}$ before the onset of transmission instability, where $z_{r_{1}}=36000$ for $\mathrm{N}=2, z_{r_{2}}=21270$ for $\mathrm{N}=3$, and $z_{r_{3}}=5300$ for $\mathrm{N}=4$. Figures 3(b), 3)(d), and 3(f) show the pulse patterns $\left|\psi_{j}(t, z)\right|$ at $z=z_{f}$, i.e., at the onset of transmission instability. As seen in Fig. 2, the soliton amplitudes tend to the equilibrium value $\eta=1$ with increasing distance for $N=2,3$, and 4 , i.e., the transmission is stable up to the distance $z=z_{r}$ in all three cases. The approach to the equilibrium state takes place along distances that are much shorter compared with the distances along which stable transmission is observed. Furthermore, the agreement between the predictions of the predator-prey model and the coupled-NLS simulations is excellent for $0 \leq z \leq z_{r}$. Additionally, as seen in Figs. 3(a), 3(c), and 3(e), the solitons retain their 
(a)

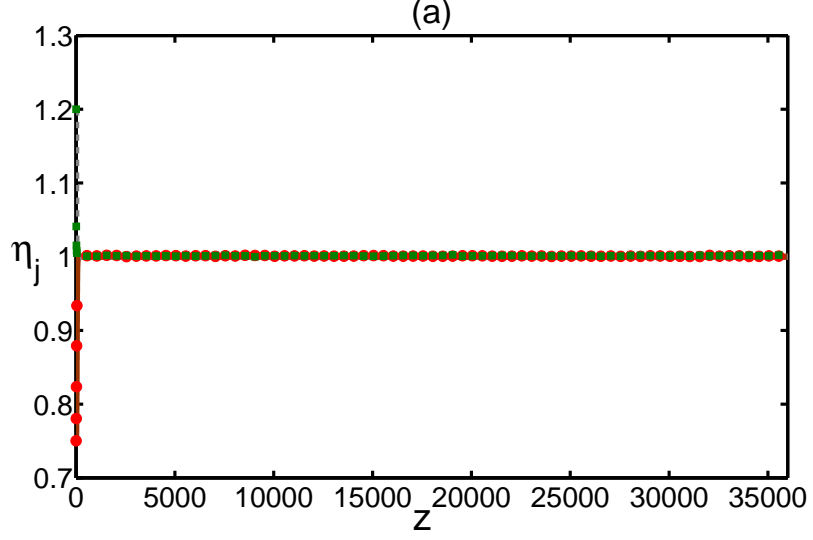

(c)

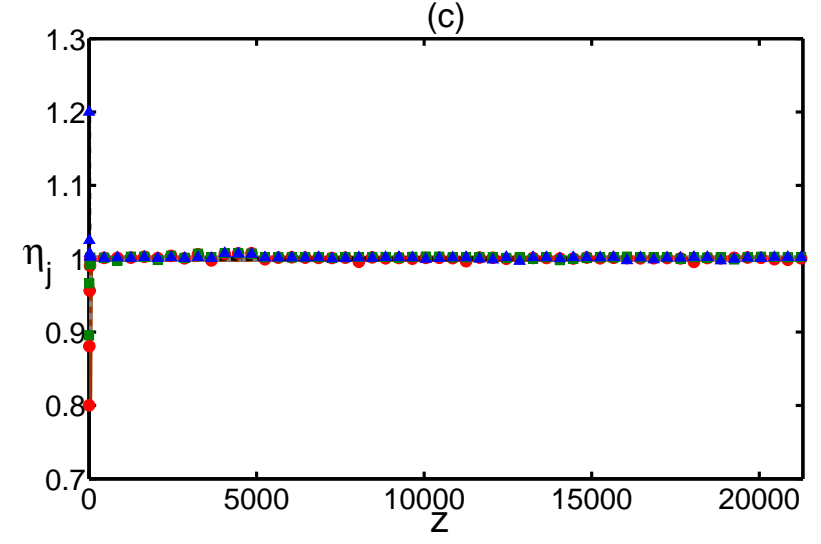

(e)

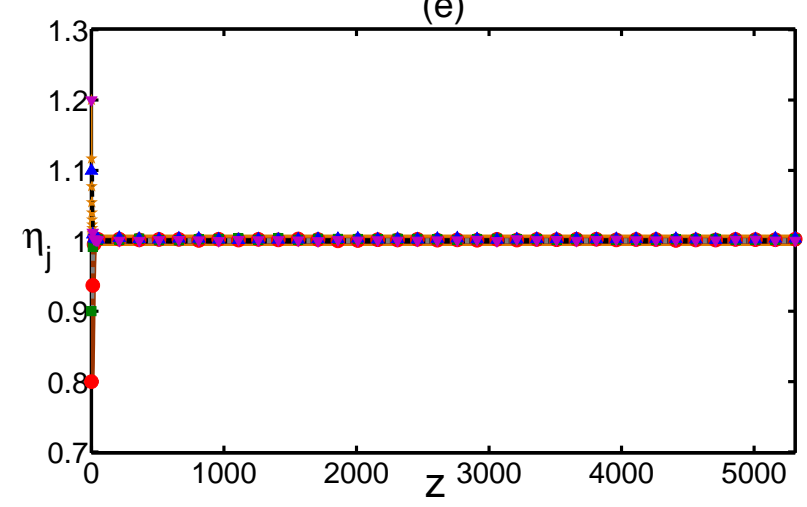

(b)

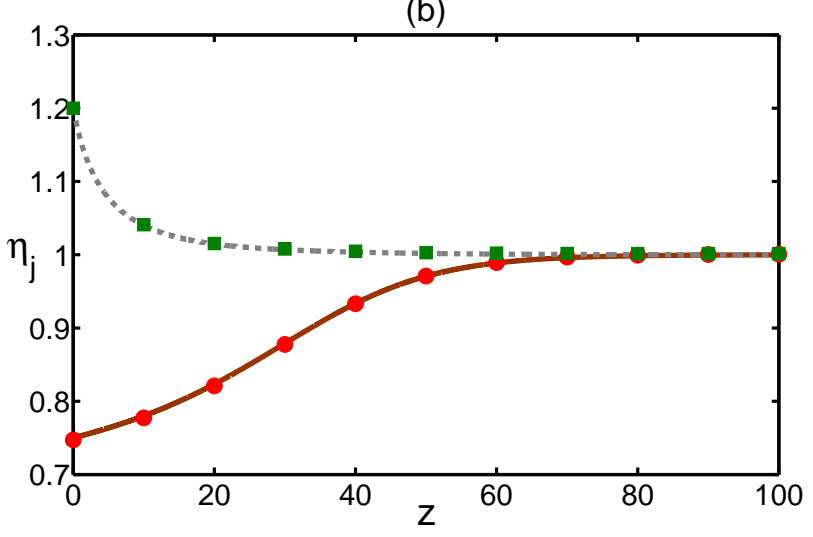

(d)

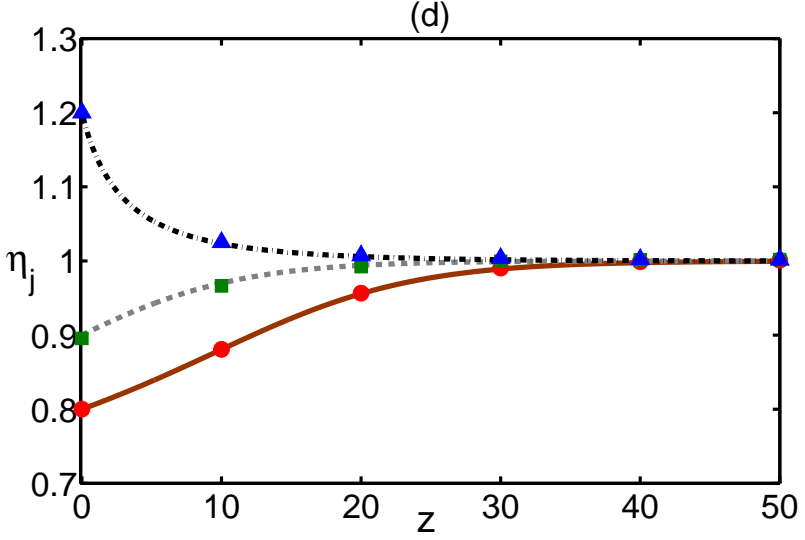

(f)

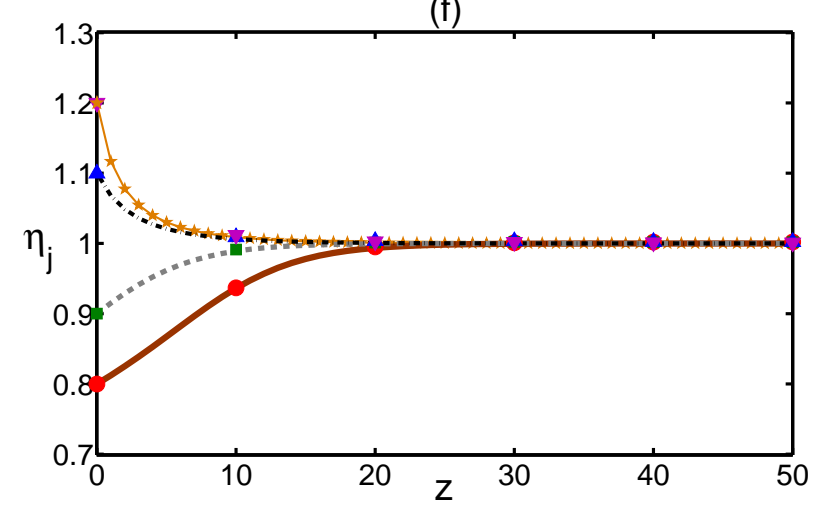

FIG. 2: The $z$ dependence of soliton amplitudes $\eta_{j}$ during transmission stabilization in waveguides with broadband delayed Raman response and narrowband GL gain-loss for two-sequence [(a) and (b)], three-sequence [(c) and (d)], and four-sequence [(e) and (f)] transmission. Graphs (b), (d), and (f) show magnified versions of the $\eta_{j}(z)$ curves in graphs (a), (c), and (e) at short distances. The red circles, green squares, blue up-pointing triangles, and magenta down-pointing triangles represent $\eta_{1}(z), \eta_{2}(z), \eta_{3}(z)$, and $\eta_{4}(z)$, obtained by numerical simulations with Eqs. (11) and (2). The solid brown, dashed gray, dashed-dotted black, and solid-starred orange curves correspond to $\eta_{1}(z), \eta_{2}(z), \eta_{3}(z)$, and $\eta_{4}(z)$, obtained by the predator-prey model (7)). 
shape at $z=z_{r}$ despite the large number of intersequence collisions. The distances $z_{r}$, along which stable propagation is observed, are significantly larger compared with those observed in other multisequence nonlinear waveguide systems. For example, the value $z_{r_{1}}=36000$ for $N=2$ is larger by a factor of 200 compared with the value obtained in waveguides with linear gain and broadband cubic loss [17]. Moreover, the stable propagation distances observed in the current work for $N=2, N=3$, and $N=4$ are larger by factors of 37.9,34.3, and 10.6 compared with the distances obtained in single-waveguide transmission in the presence of delayed Raman response and in the absence of nonlinear gain-loss [21]. The latter increase in the stable transmission distances is quite remarkable, considering the fact that in Ref. [21], intrasequence frequency-dependent linear gain-loss was employed to further stabilize the transmission, whereas in the current work, the gain-loss experienced by each sequence is uniform. We also point out that the results of our numerical simulations provide the first example for stable long-distance propagation of $N$ soliton sequences with $N>2$ in systems described by coupled GL models.

We note that at the onset of transmission instability, the pulse patterns become distorted, where the distortion appears as fast oscillations of $\left|\psi_{j}(t, z)\right|$ that are most pronounced at the solitons' tails [see Figs. 3(b), 3(d), and 3(f)]. The degree of pulse distortion is different for different pulse sequences. Indeed, for $N=2$, the $j=1$ sequence is significantly distorted at $z=z_{f_{1}}$, while no significant distortion is observed for the $j=2$ sequence. For $N=3$, the $j=1$ sequence is significantly distorted, the $j=3$ sequence is slightly distorted, while the $j=2$ sequence is still not distorted at $z=z_{f_{2}}$. For $N=4$, the $j=1$ and $j=4$ sequences are both significantly distorted at $z=z_{f_{3}}$, while no significant distortion is observed for the $j=2$ and $j=3$ sequences at this distance.

The distortion of the pulse patterns and the associated transmission destabilization can be explained by examination of the Fourier transforms of the pulse patterns $\left|\hat{\psi}_{j}(\omega, z)\right|$. Figure 4 shows the Fourier transforms $\left|\hat{\psi}_{j}(\omega, z)\right|$ at $z=z_{r}$ (before the onset of transmission instability) and at $z=z_{f}$ (at the onset of transmission instability). Figure 5 shows magnified versions of the graphs in Fig. 4 for small $\left|\hat{\psi}_{j}(\omega, z)\right|$ values. It is seen that the Fourier transforms of some of the pulse sequences develop pronounced radiative sidebands at $z=z_{f}$. Furthermore, the frequencies at which the radiative sidebands attain their maxima are related to the central frequencies $\beta_{j}(z)$ of the soliton sequences or to the frequency spacing $\Delta \beta$. The latter observation indicates that the processes leading to radiative sideband generation are 
(a)

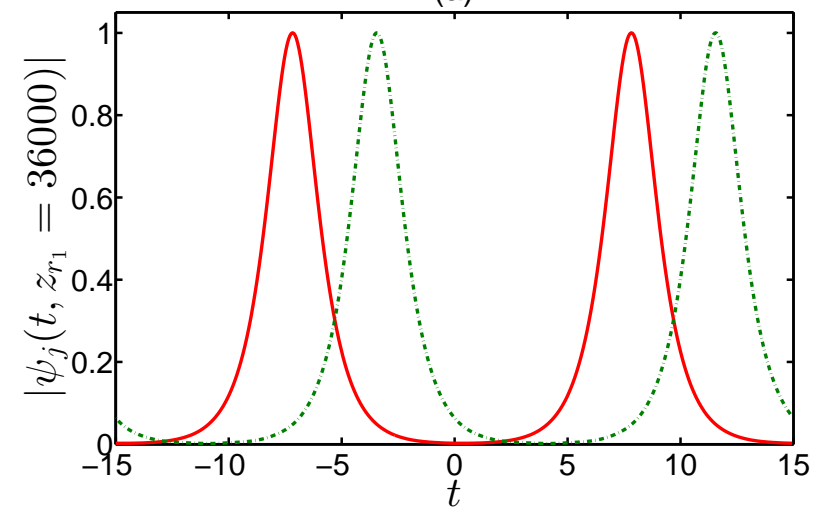

(c)

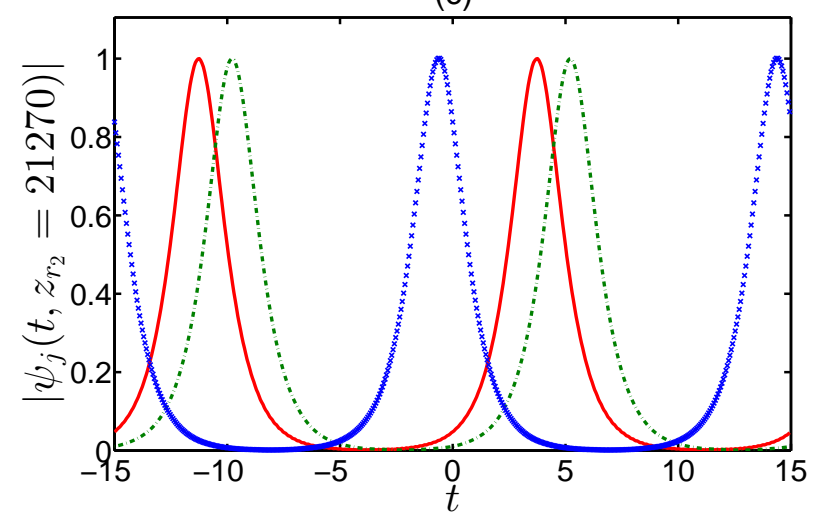

(e)

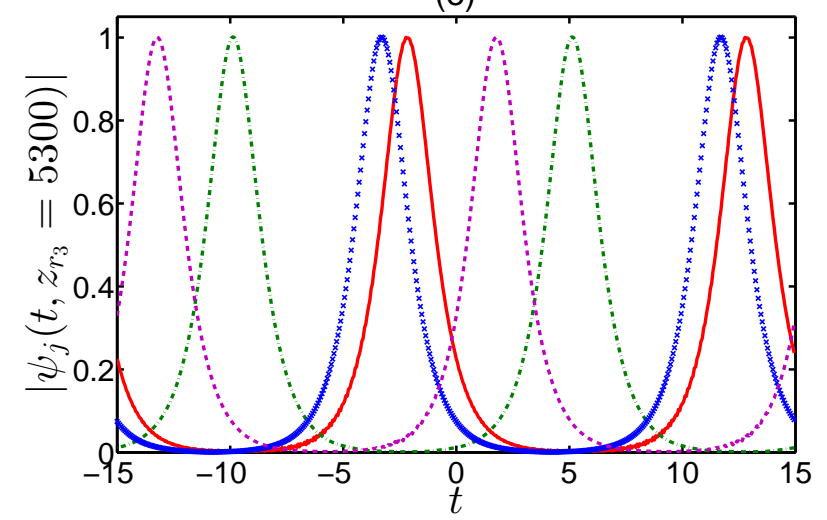

(b)

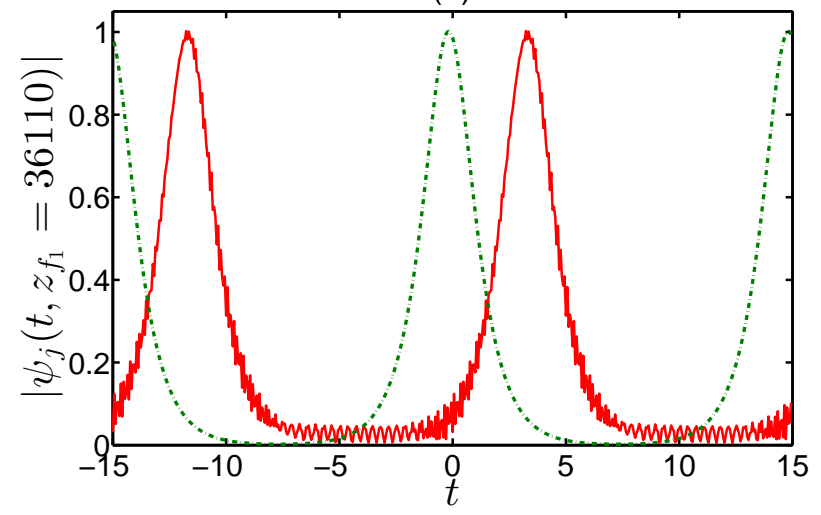

(d)

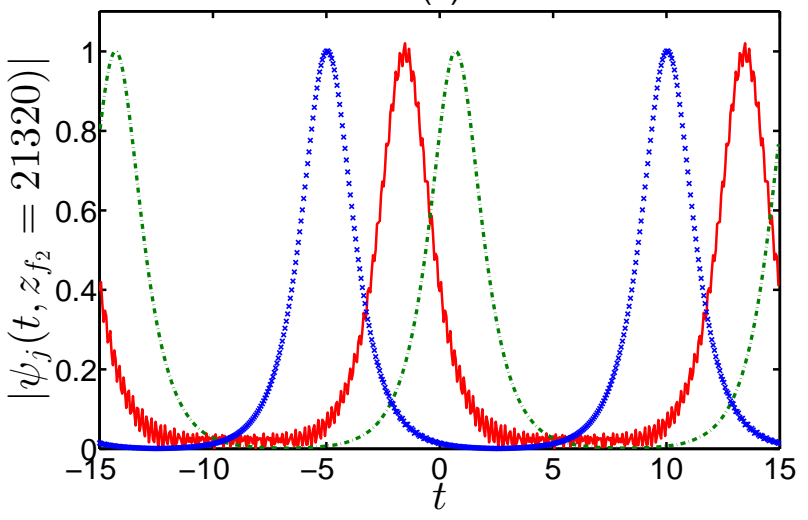

(f)

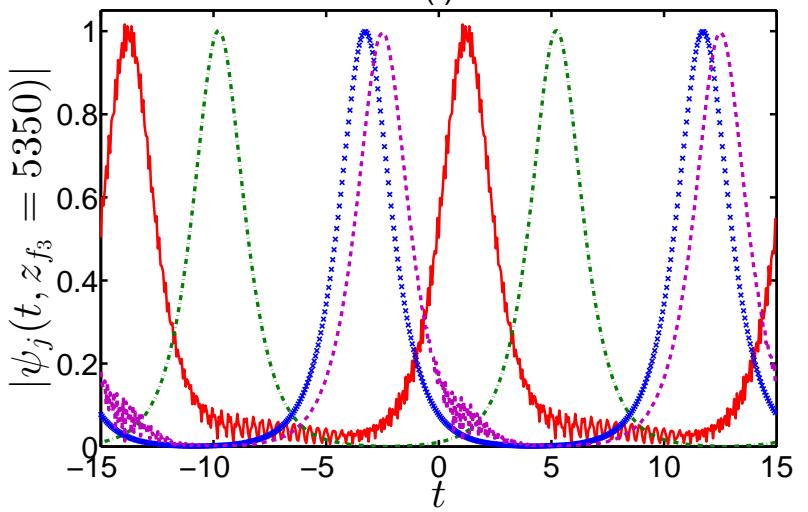

FIG. 3: The pulse patterns $\left|\psi_{j}(t, z)\right|$ near the onset of transmission instability for the two-sequence [(a) and (b)], three-sequence [(c) and (d)], and four-sequence [(e) and (f)] transmission setups considered in Fig. 2. Graphs (a), (c), and (e) show $\left|\psi_{j}(t, z)\right|$ before the onset of instability, while graphs (b), (d), and (f) show $\left|\psi_{j}(t, z)\right|$ at the onset of instability. The solid red curve, dasheddotted green curve, blue crosses, and dashed magenta curve represent $\left|\psi_{j}(t, z)\right|$ with $j=1,2,3,4$, obtained by numerical solution of Eqs. (1) and (2). The propagation distances are $z=z_{r_{1}}=36000$ (a), $z=z_{f_{1}}=36110$ (b), $z=z_{r_{2}}=21270$ (c), $z=z_{f_{2}}=21320$ (d), $z=z_{r_{3}}=5300$ (e), and $z=z_{f_{3}}=5350$ (f). 
resonant in nature (see also Refs. [21, 49]).

Consider first the Fourier transforms of the pulse patterns for $N=2$. As seen in Figs. 4(b) and 5(b), in this case the $j=1$ sequence develops radiative sidebands at frequencies $\omega_{s}^{(11)}=17.18$ and $\omega_{s}^{(12)}=34.76$ at $z=z_{f_{1}}$. In contrast, no significant sidebands are observed for the $j=2$ sequence at this distance. These findings explain the significant pulse pattern distortion of the $j=1$ sequence and the absence of pulse pattern distortion for the $j=2$ sequence at $z=z_{f_{1}}$. In addition, the radiative sideband frequencies satisfy the simple relations: $\omega_{s}^{(11)}-\beta_{2}\left(z_{r_{1}}\right) \sim 29.3 \sim 2 \Delta \beta$ and $\omega_{s}^{(12)} \sim 2 \omega_{s}^{(11)}$. For $N=3$, the $j=1$ sequence develops significant sidebands at frequencies $\omega_{s}^{(11)}=0.0$ and $\omega_{s}^{(12)}=44.4$, the $j=3$ sequence develops a weak sideband at frequency $\omega_{s}^{(31)}=-31.42$, and the $j=2$ sequence does not have any significant sidebands at $z=z_{f_{2}}$ [see Figs. 4(d) and [5(d)]. These results coincide with the significant pulse pattern distortion of the $j=1$ sequence, the weak pulse pattern distortion of the $j=3$ sequence, and the absence of pulse pattern distortion for the $j=2$ sequence at $z=z_{f_{2}}$. Additionally, the sideband frequencies satisfy the simple relations: $\omega_{s}^{(11)} \sim \beta_{3}\left(z_{r_{2}}\right), \omega_{s}^{(12)} \sim 3 \Delta \beta$, and $\omega_{s}^{(31)} \sim \beta_{1}\left(z_{r_{2}}\right)$. For $N=4$, the $j=1$ and $j=4$ sequences develop significant sidebands, while no significant sidebands are observed for the $j=2$ and $j=3$ sequences at $z=z_{f_{3}}$ [see Figs. 4(f) and 5(f)]. These findings explain the significant pulse pattern distortion of the $j=1$ and $j=4$ sequences and the absence of significant pulse pattern distortion for the $j=2$ and $j=3$ sequences at $z=z_{f_{3}}$. The sideband frequencies of the $j=1$ sequence satisfy the relations: $\omega_{s}^{(11)}=17.17 \sim \beta_{3}\left(z_{r_{3}}\right)$, and $\omega_{s}^{(12)}=34.77 \sim 2 \omega_{s}^{(11)}$. Note that the values of $\omega_{s}^{(11)}$ and $\omega_{s}^{(12)}$ for $N=4$ are very close to the values found for $N=2$. Finally, the sideband frequencies of the $j=4$ sequence satisfy the relations: $\omega_{s}^{(41)}=-27.65 \sim \beta_{1}\left(z_{r_{3}}\right)$, and $\omega_{s}^{(42)}=34.35 \sim 2 \omega_{s}^{(11)}$.

We now turn to describe numerical simulations for a single transmission switching event in waveguides with broadband delayed Raman response and a narrowband GL gain-loss profile. As described in Section III, on-off switching of $M$ out of $N$ pulse sequences at a distance $z=z_{s}$ is realized by changing the value of one or more of the physical parameters, such that the steady state $(\eta, \ldots, \eta)$ turns from asymptotically stable to unstable, while another steady state at $\left(0, \ldots, 0, \eta_{s(M+1)}, \ldots, \eta_{s N}\right)$ is asymptotically stable. We denote the on-off switching setups by A1-A2, where A1 and A2 denote the sets of physical parameters used at $0 \leq z<z_{s}$ and $z \geq z_{s}$, respectively.

Off-on switching of $M$ out of $N$ soliton sequences at $z=z_{s}$ is realized by changing the 
(a)

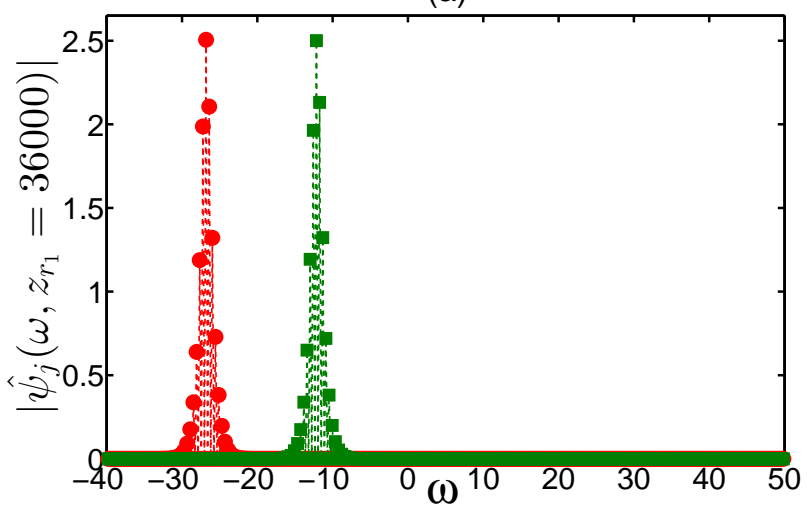

(c)

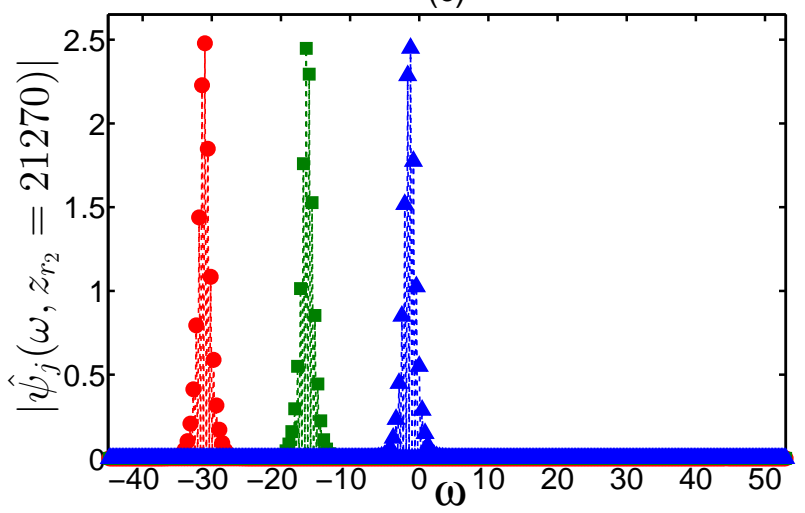

(e)

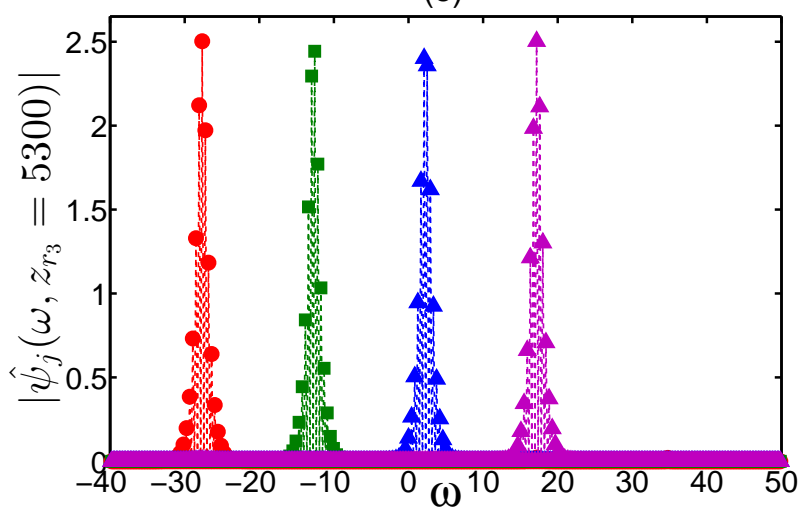

(b)

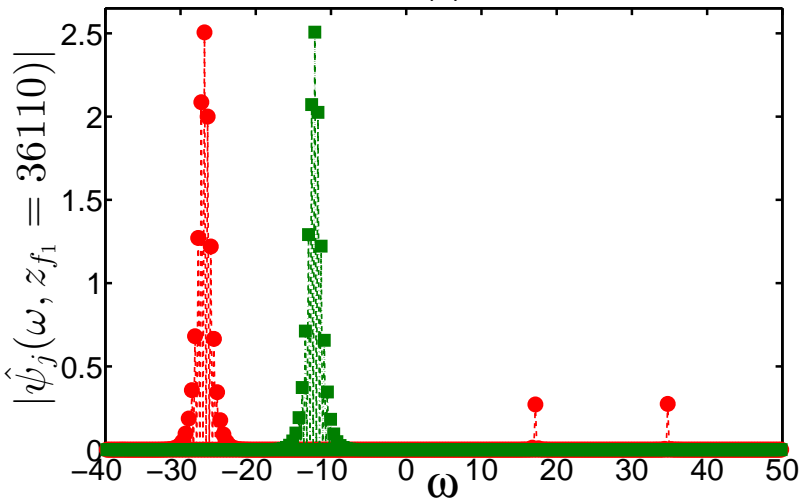

(d)

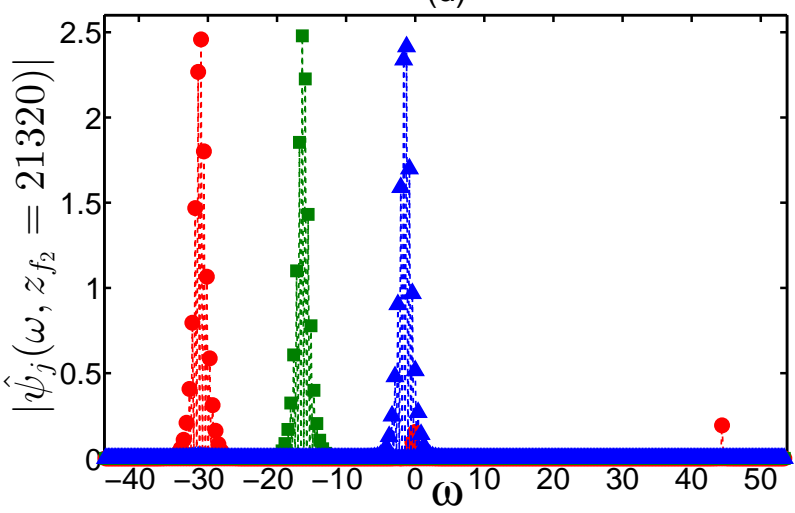

(f)

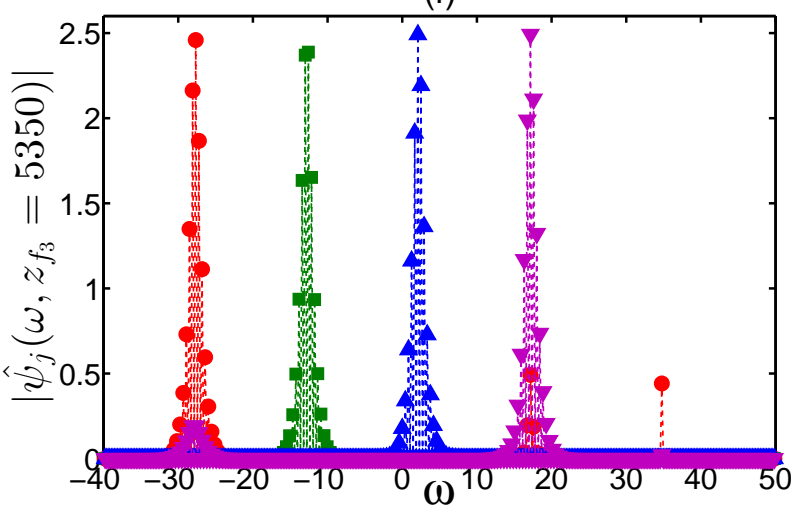

FIG. 4: The Fourier transforms of the pulse patterns $\left|\hat{\psi}_{j}(\omega, z)\right|$ near the onset of transmission instability for the two-sequence [(a) and (b)], three-sequence [(c) and (d)], and four-sequence [(e) and (f)] transmission setups considered in Figs. 22 and 3, Graphs (a), (c), and (e) show $\left|\hat{\psi}_{j}(\omega, z)\right|$ before the onset of instability, while graphs (b), (d), and (f) show $\left|\hat{\psi}_{j}(\omega, z)\right|$ at the onset of instability. The red circles, green squares, blue up-pointing triangles, and magenta down-pointing triangles represent $\left|\hat{\psi}_{j}(\omega, z)\right|$ with $j=1,2,3,4$, obtained by numerical solution of Eqs. (11) and (2). The propagation distances are $z=z_{r_{1}}=36000$ (a), $z=z_{f_{1}}=36110$ (b), $z=z_{r_{2}}=21270$ (c), $z=z_{f_{2}}=21320(\mathrm{~d}), z=z_{r_{3}}=5300(\mathrm{e})$, and $z=z_{f_{3}}=5350(\mathrm{f})$. 


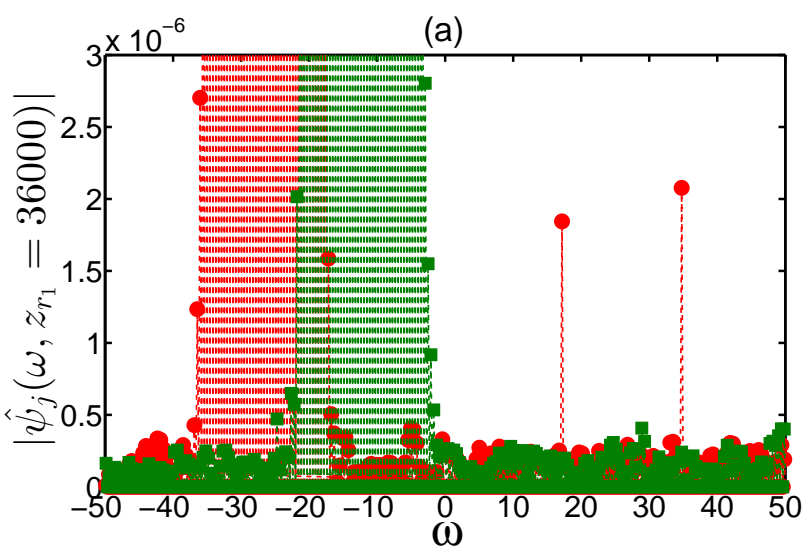

(c)

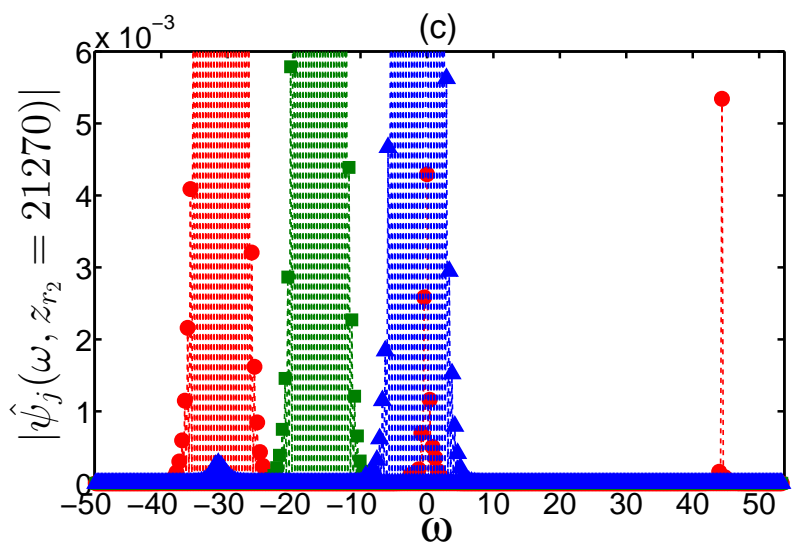

(e)

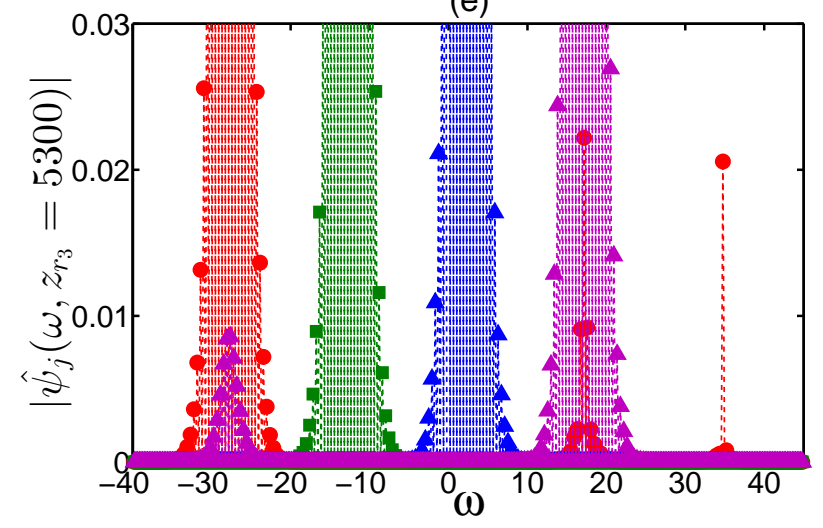

(b)

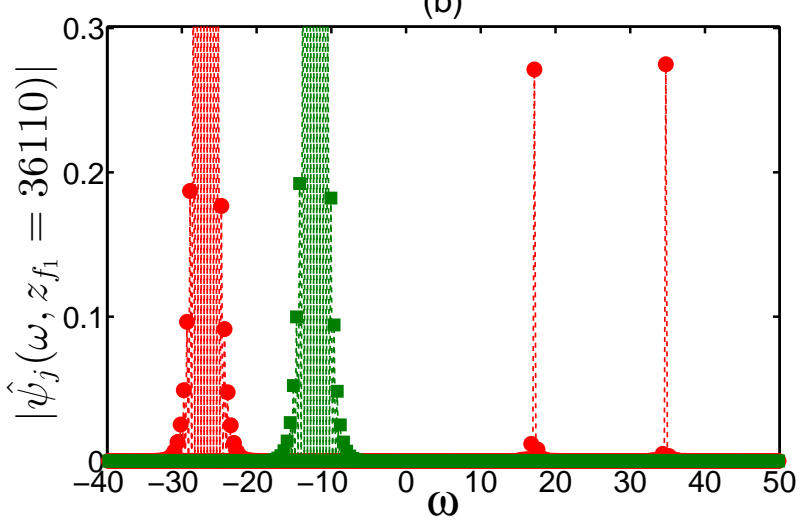

(d)

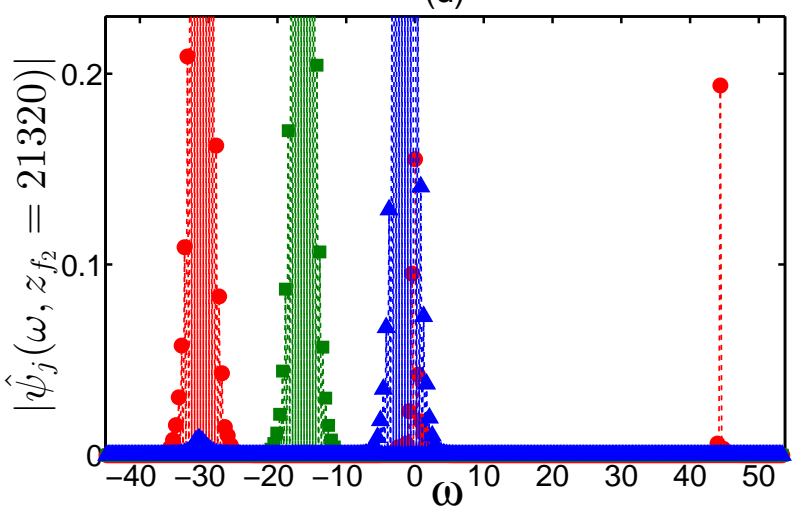

(f)

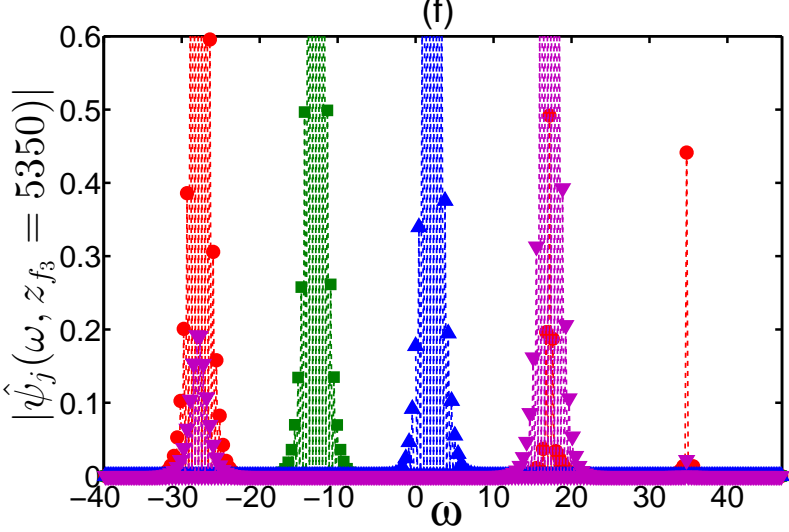

FIG. 5: Magnified versions of the graphs in Fig. 4 for small $\left|\hat{\psi}_{j}(\omega, z)\right|$ values. The symbols and distances are the same as in Fig. 4.

physical parameter values such that $(\eta, \ldots, \eta)$ turns from unstable to asymptotically stable. As explained in Section III, to achieve stable long-distance transmission after the switching, one needs to require that the origin is an asymptotically stable steady state as well. Under this requirement, $\kappa$ must satisfy inequality (12), and as a result, the basin of attraction of $(\eta, \ldots, \eta)$ is limited to $\left(\left(5 \kappa / 4-\eta^{2}\right)^{1 / 2}, \infty\right)$ for $1 \leq j \leq N$. This leads to limitations on 
the turning on of the $M$ sequences, especially for $M \geq 2$ and $N \geq 3$. To overcome this difficulty, we consider a hybrid waveguide consisting of a span with a GL gain-loss profile, a span with linear gain-loss and cubic loss, and a second span with a GL gain-loss profile. The introduction of the intermediate waveguide span with linear gain-loss and cubic loss enables the turning on of the $M$ sequences from low amplitude values due to the global stability of the steady state $(\eta, \ldots, \eta)$ for the corresponding predator-prey model. However, due to the presence of linear gain and the instability of the origin for the same predator-prey model, propagation in the waveguide span with linear gain-loss and cubic loss is unstable against emission of small amplitude waves. For this reason, we introduce the frequency dependent linear gain-loss $g(\omega, z)$ of Eq. (44) when simulating propagation in the second span. More importantly, propagation in the second span with a GL gain-loss profile leads to mitigation of radiative instability due to the presence of linear loss in all channels for this waveguide span. This enables stable long-distance propagation of the $N$ soliton sequences after the switching. We denote the off-on switching setups by A2-B-A1, where A2, B, and A1 denote the sets of physical parameters used in the first, second, and third spans of the hybrid waveguide. The first span is located at $\left[0, z_{s 1}\right)$, the second at $\left[z_{s 1}, z_{s 2}\right)$, and the third at $\left[z_{s 2}, z_{f}\right]$, where $z_{f}$ is the final propagation distance. Thus, off-on switching of the $M$ soliton sequences occurs at $z \geq z_{s 1}$, while final transmission stabilization takes place at $z \geq z_{s 2}$.

We present here the results of numerical simulations for on-off and off-on switching of two and three soliton sequences in four-sequence transmission. As discussed in the preceding paragraphs, on-off switching setups are denoted by A1-A2 and off-on switching setups are denoted by A2-B-A1. The following values of the physical parameters are used. The Raman coefficient is $\epsilon_{R}=0.0006$, which is the same value used in transmission stabilization. The other parameter values used in setup A1 in both on-off and off-on switching are $\epsilon_{5}=0.1$, $\kappa=1.2$, and $\eta=1$. The parameter values used in setup A2 in on-off switching are $\epsilon_{5}=0.04$, $\kappa=1.8$, and $\eta=1.05$ for $M=2$ and $\epsilon_{5}=0.04, \kappa=2$, and $\eta=1.1$ for $M=3$. The on-off switching distance is $z_{s}=250$ for both $M=2$ and $M=3$. The parameter values used in setup A2 in off-on switching are $\epsilon_{5}=0.032, \kappa=2.2$, and $\eta=1.1$ for $M=2$ and $\epsilon_{5}=0.02$, $\kappa=2.8$, and $\eta=1.3$ for $M=3$. The parameter values used in off-on switching in setup $\mathrm{B}$ are $\epsilon_{3}^{(2)}=0.02$ and $\eta=1$ for both $M=2$ and $M=3$. To suppress radiative instability during propagation in waveguide spans with linear gain-loss and cubic loss (setup B), the frequency dependent linear gain-loss $g(\omega, z)$ of Eq. (44) with $W=10$ and $g_{L}=-0.5$ is 
employed. The switching and final stabilization distances in off-on transmission switching are $z_{s 1}=30$ and $z_{s 2}=80$ for $M=2$, and $z_{s 1}=30$ and $z_{s 2}=90$ for $M=3$. We point out that similar results were obtained with other choices of the physical parameter values, satisfying the stability conditions discussed in Section ЏII.

The results of numerical simulations with Eqs. (11) and (2) for on-off switching of two and three soliton sequences in four-sequence transmission in setup A1-A2 are shown in Figs. [6(a) and 6(b). The results of simulations with Eqs. (1)-(4) for off-on switching of two and three sequences in four-sequence transmission in setup A2-B-A1 are shown in Figs. 6(c) and 6(d). A comparison with the predictions of the predator-prey model (77) is also presented. The agreement between the coupled-NLS simulations and the LV model's predictions is excellent in all four cases. More specifically, in on-off transmission of $M$ sequences with $M=2$ and $M=3$, the amplitudes of the solitons in the $M$ lowest frequency channels tend to zero, while the amplitudes of the solitons in the $N-M$ high frequency channels tend to new values $\eta_{s j}$, where $M+1 \leq j \leq N$. The values of the new amplitudes are $\eta_{s 3}=1.2499$ and $\eta_{s 4}=1.2878$ in on-off switching of two sequences, and $\eta_{s 4}=1.3640$ in on-off switching of three sequences. As can be seen from Figs. 6(a) and 6(b), these values are in excellent agreement with the predictions of the predator-prey model (7). In off-on switching of $M$ soliton sequences, the amplitudes of the solitons in the $M$ low frequency channels tend to zero for $z<z_{s 1}$, while the amplitudes of the solitons in the $N-M$ high frequency channels increase with $z$ for $z<z_{s 1}$. After the switching, i.e., for distances $z \geq z_{s 1}$, the amplitudes of the solitons in the $M$ low frequency channels increase to the steady-state value of 1 , while the amplitudes of the solitons in the $N-M$ high frequency channels decrease and tend to 1 , in full agreement with the predator-prey model's predictions. Note that very good agreement between the coupled-NLS and predator-prey models is observed even when some of the soliton amplitudes are small, i.e., even outside of the perturbative regime, where the predator-prey model is expected to hold. The results of the simulations presented in Fig. 66 and similar results obtained with other sets of the physical parameters demonstrate that it is indeed possible to realize stable scalable on-off and off-on transmission switching in the waveguide setups considered in the current study. Furthermore, the simulations confirm that design of the switching setups can be guided by stability and bifurcation analysis for the steady states of the predator-prey model (7). We point out that the off-on switching setups can also be employed in broadband transmission recovery, that is, in the simultaneous 
(a)

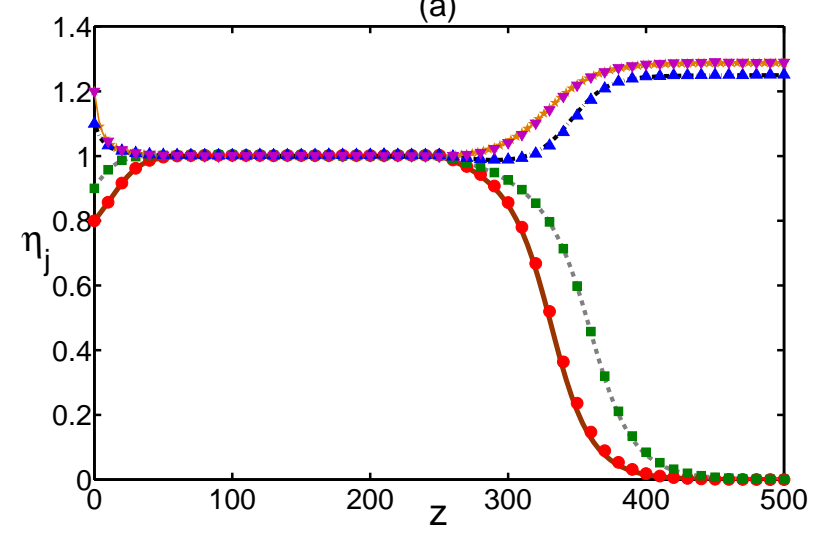

(c)

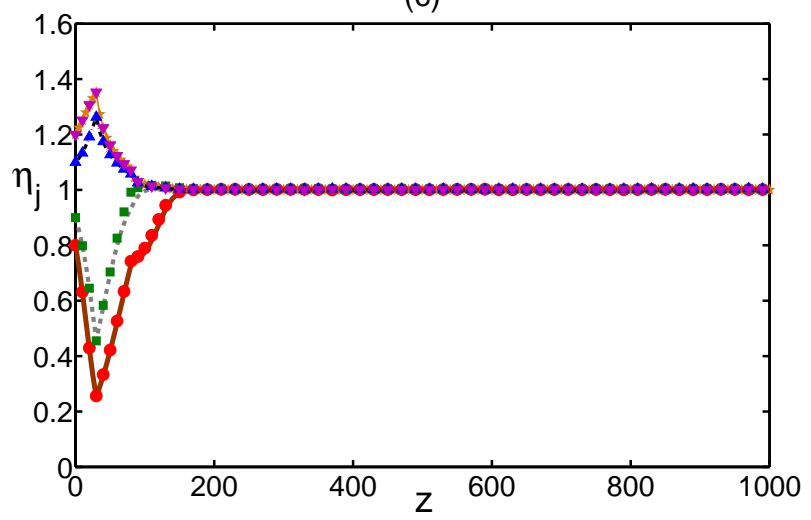

(b)

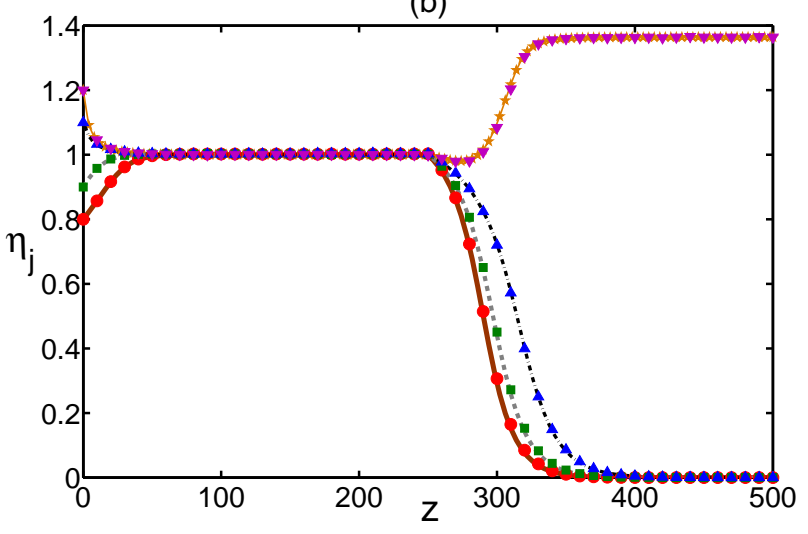

(d)

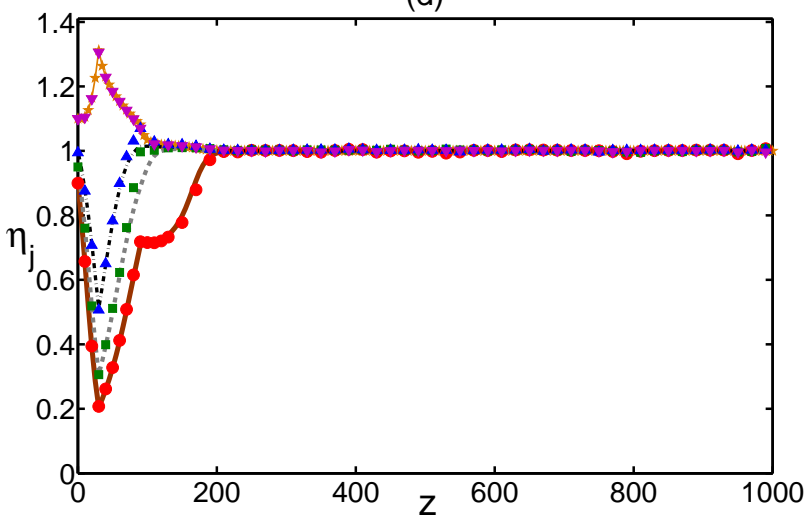

FIG. 6: The $z$ dependence of soliton amplitudes $\eta_{j}$ during single transmission switching events in four-sequence transmission in waveguides with broadband delayed Raman response and narrowband nonlinear gain-loss. (a) and (b) show on-off switching of two and three soliton sequences in waveguides with a GL gain-loss profile in setup A1-A2, while (c) and (d) show off-on switching of two and three sequences in hybrid waveguides in setup A2-B-A1. The red circles, green squares, blue up-pointing triangles, and magenta down-pointing triangles represent $\eta_{1}(z), \eta_{2}(z), \eta_{3}(z)$, and $\eta_{4}(z)$, obtained by numerical simulations with Eqs. (11) and (2) in (a) and (b), and with Eqs. (11)-(41) in (c) and (d). The solid brown, dashed gray, dashed-dotted black, and solid-starred orange curves correspond to $\eta_{1}(z), \eta_{2}(z), \eta_{3}(z)$, and $\eta_{4}(z)$, obtained by the predator-prey model (7)).

amplification of multiple soliton sequences, which experienced significant energy decay, to a desired steady-state amplitude value.

As discussed in Section III, an important application of the switching setups considered in our paper is for realizing efficient signal processing in multichannel transmission. In such application, the amplitude values $\eta_{j}$ are used to encode information about the type of signal processing to be carried out in the next processing station. As a result, the pulse sequences 
typically undergo multiple switching events and it is important to show that this can be realized in a stable manner. We therefore turn to discuss the results of numerical simulations with the coupled-NLS model (11) for multiple switching events. As a specific example, we consider multiple switching in a three-channel system in the hybrid waveguide setup A1(A2-B-A1)-...-(A2-B-A1), where (A2-B-A1) repeats six times. Thus, in this case the soliton sequences first experience transmission stabilization in waveguide setup A1, and then undergo six successive off-on switching events in waveguide setup A2-B-A1. The parameter values are chosen such that during on switching, amplitude values in all sequences tend to 1 , while during off switching, transmission of a single sequence (the lowest-frequency sequence $j=1$ ) is turned off. We emphasize, however, that similar results are obtained with other numbers of channels and in other switching scenarios. For the example presented here, during on switching stabilization in waveguide setup A1, $\epsilon_{R}=0.0006, \epsilon_{5}=0.15, \kappa=1.2$, and $\eta=1$ are used, while during off switching in waveguide setup A2, $\epsilon_{R}=0.0024, \epsilon_{5}=0.024$, $\kappa=2.2$, and $\eta=1.15$ are used. Note that the higher value of $\epsilon_{R}$ in waveguide setup A2 is required for realizing a faster on-off transmission switching, that is, for decreasing the distance along which the off switching takes place. Additionally, during on switching in waveguide $\operatorname{setup} \mathrm{B}, \epsilon_{R}=0.0006, \epsilon_{3}^{(2)}=0.02$, and $\eta=1$ are chosen. To suppress radiative instability during propagation in waveguide spans with linear gain-loss and cubic loss (setup B), the frequency dependent linear gain-loss $g(\omega, z)$ of Eq. (44) with $W=10$ and $g_{L}=-0.5$ is employed. In the simulations, transmission of the $j=1$ sequence is turned off in setup A2 at distances $z_{3 m+1}=700(m+1)$ for $0 \leq m \leq 5$. Transmission of sequence $j=1$ is turned on in waveguide setup B at $z_{3 m+2}=50+700(m+1)$ for $0 \leq m \leq 5$, and transmission stabilization in setup A1 starts at $z_{0}=0$ and at $z_{3 m}=100+700 m$ for $1 \leq m \leq 6$. The final propagation distance is $z_{f}=5000$. Thus, the waveguide spans are $[0,700),[700,750)$, $[750,800),[800,1400), \ldots,[4200,4250),[4250,4300)$, and $[4300,5000]$.

The results of numerical simulations with Eqs. (1)-(41) for multiple transmission switching events are shown in Fig. 7 along with the predictions of the predator-prey model (7). The agreement between the coupled-NLS simulations and the predator-prey model's predictions is excellent throughout the propagation. Furthermore, as seen in Fig. 7(b), no pulse distortion is observed at the final propagation distance $z_{f}$. Note that the minimal values of $\eta_{1}(z)$, which are attained prior to the start of the on switch, are $\eta_{1}\left(z_{3 m+2}\right)=0.33$. As a result, the value of the decision level $\eta_{t h}$ for distinguishing between on and off transmission 
states can be set as low as $\eta_{t h}=0.35$, which is significantly lower than the value $\eta_{t h}=0.65$ obtained in Ref. [19] for transmission in a two-channel waveguide system with a broadband GL gain-loss profile. The results presented in Fig. 7 along with results of numerical simulations with other sets of the physical parameter values demonstrate that stable multiple transmission switching events can indeed be realized over a wide range of amplitude values, using waveguides with broadband delayed Raman response and narrowband nonlinear gain-loss.

\section{DISCUSSION}

Let us discuss the reasons for the robustness and scalability of transmission stabilization and switchng in waveguides with broadband delayed Raman response and narrowband nonlinear gain-loss. The scalability and robustness of transmission control can be attributed to the following properties of these waveguides. (1) The asymptotic stability of the steady state $(\eta, \ldots, \eta)$ for waveguides with a GL gain-loss profile, which is independent of $N$ and $\epsilon_{R}$, is key to realizing scalable transmission stabilization and switching. (2) The presence of net linear loss in all frequency channels for waveguides with a GL gain-loss profile leads to mitigation of radiative instability. (3) Due to the narrow bandwidth of the nonlinear gain-loss, three-pulse interaction does not contribute to collision-induced amplitude shifts. As a result, the extension of the predator-prey model from $N=2$ to a general $N$ value is straightforward. This also makes the extension of waveguide setup design from $N=2$ to a general $N$ value straightforward. In contrast, in waveguides with broadband nonlinear gain-loss, three-pulse interaction gives an important contribution to collision-induced amplitude shifts [18, 34]. Due to the complex nature of three-pulse interaction in generic three-soliton collisions in waveguides with broadband nonlinear gain or loss (see Ref. [34]), it is very difficult to extend the LV model for amplitude dynamics from $N=2$ to a generic $N$ value in these waveguides. In the absence of an $N$-dimensional $L V$ model, it is unclear how to design setups for stable transmission stabilization and switching in $N$-sequence waveguide systems with broadband nonlinear gain-loss. As a result, transmission stabilization and switching in waveguides with broadband nonlinear gain-loss have been so far limited to two-sequence systems [18 20]. (4) The Raman-induced energy transfer in soliton collisions from high-frequency solitons to low-frequency solitons is an important ingerdient in 
(a)

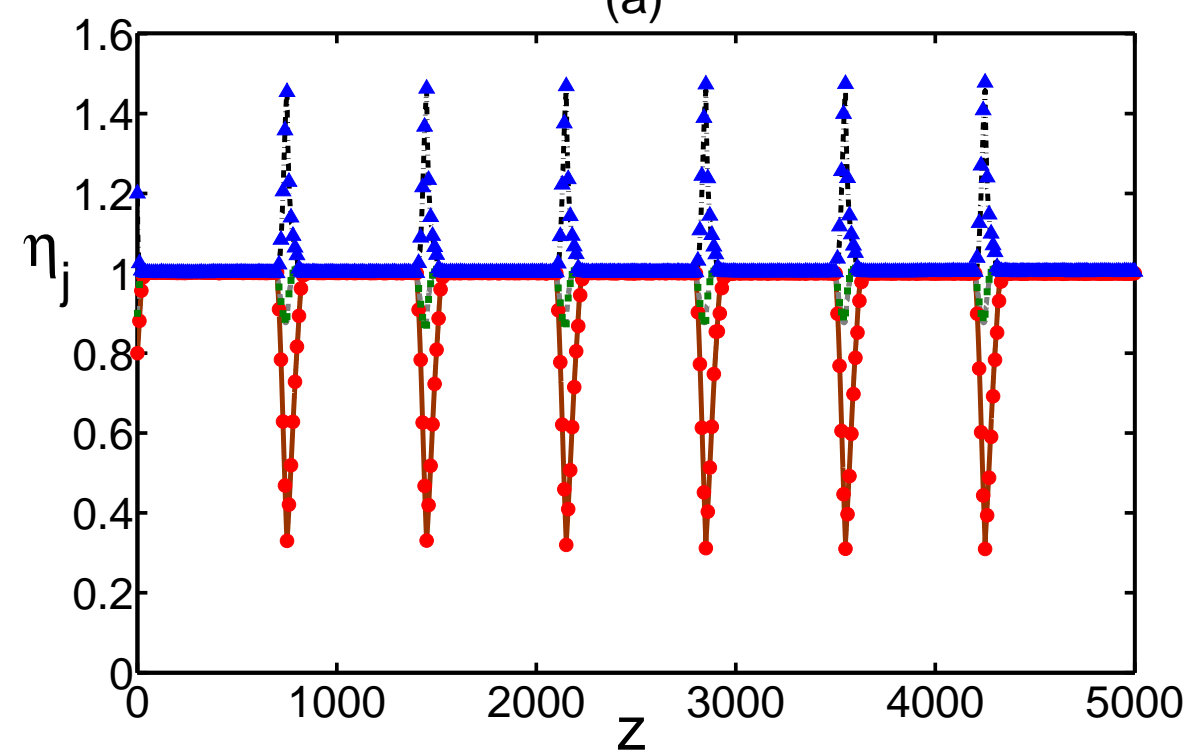

(b)

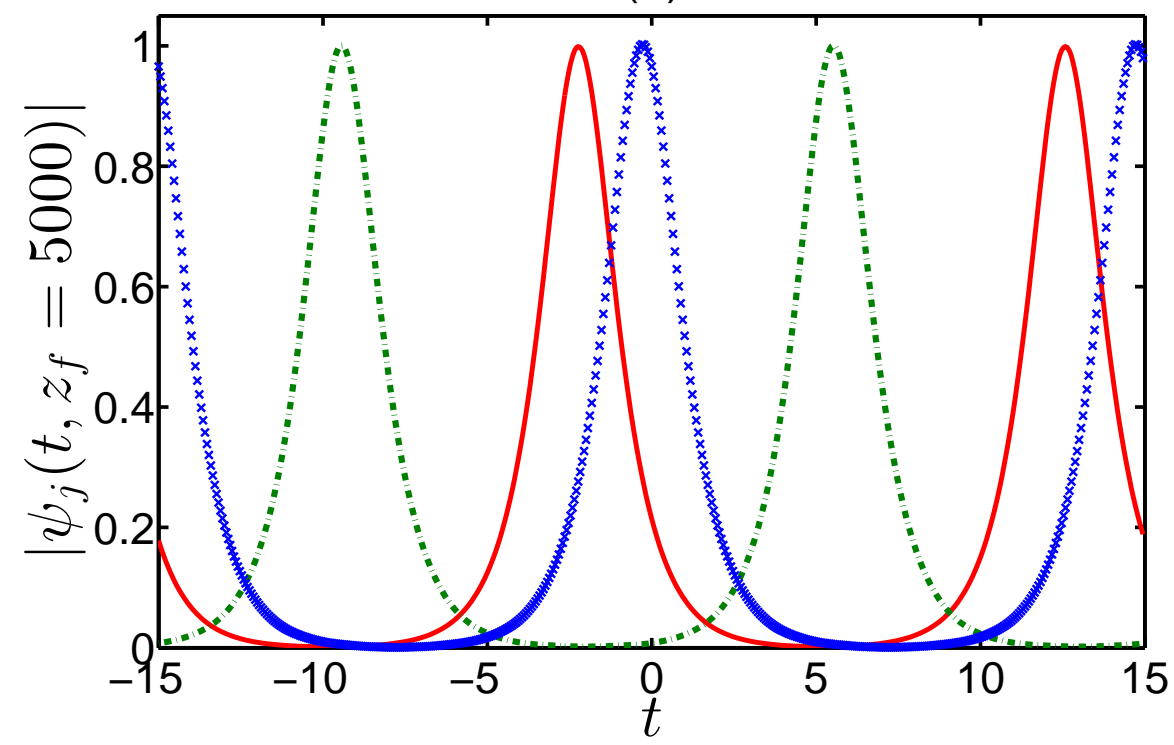

FIG. 7: (a) The $z$ dependence of soliton amplitudes $\eta_{j}$ in multiple transmission switching with three sequences $(N=3)$ in hybrid waveguide setup A1-(A2-B-A1)-...-(A2-B-A1), where (A2-B-A1) repeats six times. In this case, the soliton sequences undergo transmission stabilization followed by six successive off-on switching events. The red circles, green squares, and blue up-pointing triangles represent $\eta_{1}(z), \eta_{2}(z)$, and $\eta_{3}(z)$, obtained by numerical simulations with Eqs. (11)-(41). The solid brown, dashed gray, and dashed-dotted black curves correspond to $\eta_{1}(z), \eta_{2}(z)$, and $\eta_{3}(z)$, obtained by the predator-prey model (7). (b) The pulse patterns $\left|\psi_{j}(t, z)\right|$ at the final distance $z=z_{f}=5000$, as obtained by numerical solution of Eqs. (11)-(4). The solid red curve, dashed-dotted green curve, and blue crosses represent $\left|\psi_{j}(t, z)\right|$ with $j=1,2,3$. 
the realization of scalable on-off switching. Indeed, to compensate for the Raman-induced energy loss or gain in the collisions, high-frequency sequences should be overamplified while low-frequency sequences should be underamplified compared to mid-frequency sequences. As a result, the magnitude of the net linear loss is largest for the low-frequency sequences, and therefore, on-off switching is easiest to realize for these sequences. Thus, the presence of broadband delayed Raman response introduces a preference for turning off the transmission of the low-frequency sequences, and by this enables systematic scalable on-off switching. (5) The global asymptotic stability of the steady state $(\eta, \ldots, \eta)$ for waveguide spans with linear gain-loss and cubic loss, which is independent of all physical parameters, is important for realizing robust scalable off-on switching in hybrid waveguides. These five waveguide properties are explained by stability and bifurcation analysis for the steady states of the generalized $N$-dimensional predator-prey model for amplitude dynamics. Thus, the analysis of the predator-prey model is essential to the design of waveguide setups leading to stable scalable control of soliton-based multichannel transmission.

Note that waveguide setups with narrowband cubic gain and quinitc loss or with narrowband cubic loss can be realized by employing fast saturable absorbers with a bandwidth $\Delta \nu_{G L}$ satisfying: $\nu_{0} \ll \Delta \nu_{G L} \ll \Delta \nu$. That is, in these waveguide systems, the bandwidth of the saturable absorber is larger than the spectral width of the optical pulses but smaller than the frequency spacing between adjacent frequency channels. Waveguide systems containing a fast saturable absorber with a finite spectral width, which is much larger than the spectral width of the optical pulses, have been studied extensively in the context of mode-locked lasers; see, for example, Refs. [50 52] and references therein.

\section{CONCLUSIONS}

We developed a method for achieving stable scalable control of propagation of multiple soliton sequences in broadband optical waveguide systems. The method is based on employing nonlinear waveguides with broadband delayed Raman response, linear gain-loss, and narrowband nonlinear gain-loss. We showed that the combination of Raman-induced amplitude shifts in interchannel collisions and single-pulse amplitude shifts due to linear and nonlinear gain-loss with properly chosen physical parameter values can be used to realize

robust scalable transmission stabilization and switching. For this purpose, we first showed 
that the dynamics of soliton amplitudes in an $N$-sequence transmission system can be described by a generalized $N$-dimensional predator-prey model. We then carried out stability and bifurcation analysis for the steady states of the predator-prey model for two central cases of the gain-loss: (1) a GL gain-loss profile, (2) linear gain-loss and cubic loss. The stability and bifurcation analysis was then used to develop waveguide setups that lead to robust transmission stabilization as well as on-off and off-on switching of $M$ out of $N$ soliton sequences.

For waveguides with a GL gain-loss profile, we obtained the Lyapunov function $V_{L}(\boldsymbol{\eta})$ for the predator-prey model and used it to derive simple conditions for asymptotic stability and instability of the steady state with equal amplitudes for all sequences $(\eta, \ldots, \eta)$. These conditions are independent of the number of channels $N$ and the value of the Raman coefficient $\epsilon_{R}$, which is essential for the realization of scalable transmission stabilization and switching. We also found that the steady state at the origin is asymptotically stable, provided all the linear gain-loss coefficients are negative. Combining the requirements for asymptotic stability of both $(\eta, \ldots, \eta)$ and the origin, we showed that the smallest value of the quintic loss coefficient $\epsilon_{5}$ required for robust transmission stabilization and off-on switching for $N \gg 1$ scales as $\epsilon_{5} \sim N^{2} \epsilon_{R}$. The realization of on-off switching requires stability analysis of steady states, for which $M$ components are equal to zero. We first gave a simple argument, showing that switching off of $M$ sequences is most conveniently realized by turning off the transmission of the low-frequency sequences $1 \leq j \leq M$. We therefore focused attention on the steady state $\left(0, \ldots, 0, \eta_{s(M+1)}, \ldots, \eta_{s N}\right)$ and showed that for $N \gg 1$, stability of this steady state can be established by calculating only $N-M+1$ diagonal elements of the corresponding Jacobian matrix.

Stability analysis for the predator-prey model, describing amplitude dynamics in waveguides with linear gain-loss and cubic loss, was carried out in a similar manner. More specifically, we found that the same $V_{L}(\boldsymbol{\eta})$ that was used for the predator-prey model with a GL gain-loss profile is a Lyapunov function for the predator-prey model with linear gain-loss and cubic loss [39]. Moreover, we used this Lyapunov function to show that $(\eta, \ldots, \eta)$ is globally asymptotically stable, regardless of the values of all physical parameters. However, linear stability analysis showed that the origin is unstable in this case. The latter instability eventually leads to growth of small amplitude waves, and thus makes waveguides with linear gain-loss and cubic loss unsuitable for long-distance transmission stabilization. On the 
other hand, the global asymptotic stability of $(\eta, \ldots, \eta)$ means that waveguide spans with linear gain-loss and cubic loss can be used in hybrid waveguides for realizing robust off-on transmission switching.

The predictions of the generalized predator-prey model for scalable transmission stabilization and switching were tested by numerical simulations with a perturbed coupled-NLS model, which takes into account broadband delayed Raman response and a narrowband GL gain-loss profile. The coupled-NLS simulations for transmission stabilization were carried out with $2 \leq N \leq 4$ soliton sequences. The simulations showed stable propagation and excellent agreement with the predictions of the predator-prey model over significantly larger distances compared with those obtained in earlier works with other waveguide setups. More specifically, the stable propagation distances obtained for two-, three-, and four-sequence transmission were larger by factors of 37.9, 34.3, and 10.6, respectively, compared with the distances obtained in single-waveguide transmission in the presence of delayed Raman response and in the absence of nonlinear gain-loss [21]. Furthermore, the distance along which stable transmission was observed in a two-channel system was larger by a factor of 200 compared with the distance achieved in waveguides with linear gain and broadband cubic loss [17]. The enhanced stability of $N$-channel transmission through waveguides with broadband delayed Raman response and narrowband GL gain-loss profile was explained in the Discussion.

We demonstrated single and multiple transmission switching events of $M$ out of $N$ pulse sequences by carrying out numerical simulations with the coupled-NLS model that was described in the preceding paragraph. As examples, we presented the results of the simulations for the following setups: (a) single on-off and off-on switching events of two and three soliton sequences in four-sequence transmission; (b) six switching events in a three-sequence system, in which transmission of one soliton sequence was switched off and then on six consecutive times. The results of the coupled-NLS simulations were in excellent agreement with the predictions of the predator-prey model for both single and multiple switching events. Furthermore, the agreement was observed even when amplitude values were small for some soliton sequences, i.e., even outside of the regime where the predator-prey model's description was expected to hold. Based on these results and results of simulations with other sets of the physical parameter values, we concluded that stable scalable transmission switching can indeed be realized in waveguides with broadband delayed Raman response and narrowband 
nonlinear gain-loss.

\section{Acknowledgments}

We are grateful to T.P. Tran for help with the numerical code in the initial stages of this work. Q.M.N. and T.T.H are supported by the Vietnam National Foundation for Science and Technology Development (NAFOSTED) under Grant No. 101.99-2015.29.

\section{Author contribution statement}

A.P. initiated the project, participated in the derivation of the analytic results, and took part in the analysis of the results of numerical simulations. Q.M.N. and T.T.H carried out the numerical simulations, participated in the derivation of the analytic results, and took part in the analysis of the results of numerical simulations.

[1] G.P. Agrawal, Nonlinear Fiber Optics (Academic, San Diego, CA, 2001).

[2] F. Forghieri, R.W. Tkach, and A.R. Chraplyvy, in Optical Fiber Telecommunications III, I.P. Kaminow and T.L. Koch, eds., (Academic, San Diego, CA, 1997), Chapter 8.

[3] L.F. Mollenauer and J.P. Gordon, Solitons in Optical Fibers: Fundamentals and Applications (Academic, San Diego, CA, 2006).

[4] A.H. Gnauck, R.W. Tkach, A.R. Chraplyvy, and T. Li, J. Lightwave Technol. 26, 1032 (2008).

[5] R.-J. Essiambre, G. Kramer, P.J. Winzer, G.J. Foschini, and B. Goebel, J. Lightwave Technol. 28, $662(2010)$.

[6] For this reason, we use the equivalent terms multichannel transmission, multisequence transmission, and WDM transmission to describe the simultaneous propagation of multiple pulse sequences with different central frequencies through the same optical waveguide.

[7] Q. Lin, O.J. Painter, and G.P. Agrawal, Opt. Express 15, 16604 (2007).

[8] R. Dekker, N. Usechak, M. Först, and A. Driessen, J. Phys. D 40, R249 (2007).

[9] M.A. Foster, A.C. Turner, M. Lipson, and A.L. Gaeta, Opt. Express 16, 1300 (2008).

[10] J. Chow, G. Town, B. Eggleton, M. Ibsen, K. Sugden, and I. Bennion, IEEE Photon. Technol. Lett. 8, 60 (1996). 
[11] H. Shi, J. Finlay, G.A. Alphonse, J.C. Connolly, and P.J. Delfyett, IEEE Photon. Technol. Lett. 9, 1439 (1997).

[12] H. Zhang, D.Y. Tang, X. Wu, and L.M. Zhao, Opt. Express 17, 12692 (2009).

[13] X.M. Liu, D.D. Han, Z.P. Sun, C. Zeng, H. Lu, D. Mao, Y.D. Cui, and F.Q. Wang, Sci. Rep. 3, 2718 (2013).

[14] E. Iannone, F. Matera, A. Mecozzi, and M. Settembre, Nonlinear Optical Communication Networks (Wiley, New York, 1998).

[15] L.F. Mollenauer and P.V. Mamyshev, IEEE J. Quantum Electron. 34, 2089 (1998).

[16] Q.M. Nguyen and A. Peleg, Opt. Commun. 283, 3500 (2010).

[17] A. Peleg, Q.M. Nguyen, and Y. Chung, Phys. Rev. A 82, 053830 (2010).

[18] A. Peleg and Y. Chung, Phys. Rev. A 85, 063828 (2012).

[19] D. Chakraborty, A. Peleg, and J.-H. Jung, Phys. Rev. A 88, 023845 (2013).

[20] Q.M. Nguyen, A. Peleg, and T.P. Tran, Phys. Rev. A 91, 013839 (2015).

[21] A. Peleg, Q.M. Nguyen, and T.P. Tran, Opt. Commun. 380, 41 (2016).

[22] A.R. Chraplyvy, Electron. Lett. 20, 58 (1984).

[23] F. Forghieri, R.W. Tkach, and A.R. Chraplyvy, IEEE Photon. Technol. Lett. 7, 101 (1995).

[24] K.-P. Ho, J. Lightwave Technol. 18, 915 (2000).

[25] T. Yamamoto and S. Norimatsu, J. Lightwave Technol. 21, 2229 (2003).

[26] A. Peleg, Opt. Lett. 29, 1980 (2004).

[27] A. Peleg, Phys. Lett. A 360, 533 (2007).

[28] Y. Chung and A. Peleg, Phys. Rev. A 77, 063835 (2008).

[29] B. Bakhshi, L. Richardson, and E.A. Golovchenko, in Proceedings of the Optical Fiber Communication Conference, San Diego, CA, 2009, paper OThC4.

[30] A. Peleg and Y. Chung, Opt. Commun. 285, 1429 (2012).

[31] Raman Amplifiers for Telecommunications 1: Physical Principles, edited by M.N. Islam (Springer, New York, 2004).

[32] Raman Amplification in Fiber Optical Communication Systems, edited by C. Headley and G.P. Agrawal (Elsevier, San Diego, CA, 2005).

[33] Y. Okawachi, O. Kuzucu, M.A. Foster, R. Salem, A.C. Turner-Foster, A. Biberman, N. Ophir, K. Bergman, M. Lipson, and A.L. Gaeta, IEEE Photon. Technol. Lett. 24, 185 (2012).

[34] A. Peleg, Q.M. Nguyen, and P. Glenn, Phys. Rev. E 89, 043201 (2014). 
[35] Note that a similar approach for mitigation of radiative instability was employed in Ref. [21] for soliton propagation in the presence of delayed Raman response in the absence of nonlinear gain-loss.

[36] A.J. Lotka, Elements of Physical Biology (Williams and Wilkins, Baltimore, 1925).

[37] V. Volterra, J. Cons. Int. Explor. Mer 3, 1 (1928).

[38] Channel switching can also be implemented in amplitude-keyed transmission. In this case, one should define a second threshold level $\eta_{t h 2}$, satisfying $0<\eta_{t h 2}<\eta_{t h}$. The larger decision level $\eta_{t h}$ is then used to determine the transmission state of each channel for channel switching, while the smaller decision level $\eta_{t h 2}$ is used to determine the state of each time-slot within a given channel. Thus, in this case, the on and off states for the $j$ th channel are determined by the conditions $\eta_{j}>\eta_{t h}$ and $\eta_{t h 2}<\eta_{j}<\eta_{t h}$, respectively, where $\eta_{j}$ is the common amplitude value for pulses in occupied time slots in the $j$ th channel.

[39] It is possible to show that $V_{L}(\boldsymbol{\eta})$ of Eq. (8) is a Lyapunov function for the predator-prey model (7) even for an $m$ th-order polynomial $L$ with a negative coefficient for the $m$ th-order term and properly chosen values for the other polynomial coefficients.

[40] Linear stability analysis shows that $(\eta, \ldots, \eta)$ is a stable focus when $0<\kappa<8 \eta^{2} / 5$ and an unstable focus when $\kappa>8 \eta^{2} / 5$.

[41] Here we use the fact that the origin is a stable node of Eq. (7), so that $g_{j}<0$ for $1 \leq j \leq N$.

[42] These conditions should be augmented by the condition for asymptotic stability of $\left(0, \ldots, 0, \eta_{s N}\right)$.

[43] S. Chi and S. Wen, Opt. Lett. 14, 1216 (1989).

[44] B.A. Malomed, Phys. Rev. A 44, 1412 (1991).

[45] S. Kumar, Opt. Lett. 23, 1450 (1998).

[46] T.I. Lakoba and D.J. Kaup, Opt. Lett. 24, 808 (1999).

[47] Y. Chung and A. Peleg, Nonlinearity 18, 1555 (2005).

[48] Q.M. Nguyen and A. Peleg, J. Opt. Soc. Am. B 27, 1985 (2010).

[49] D. Chakraborty, A. Peleg, and Q.M. Nguyen, Opt. Commun. 371, 252 (2016).

[50] H.A. Haus, J. Appl. Phys. 46, 3049 (1975).

[51] J.D. Moores, Opt. Commun. 96, 65 (1993).

[52] H.A. Haus, IEEE J. Sel. Top. Quantum Electron. 6, 1173 (2000). 